\title{
Review Article \\ Topological Order: From Long-Range Entangled Quantum Matter to a Unified Origin of Light and Electrons
}

\author{
Xiao-Gang Wen ${ }^{1,2,3}$ \\ ${ }^{1}$ Perimeter Institute for Theoretical Physics, Waterloo, ON, Canada N2L 2 Y5 \\ ${ }^{2}$ Department of Physics, Massachusetts Institute of Technology, Cambridge, MA 02139, USA \\ ${ }^{3}$ Institute for Advanced Study, Tsinghua University, Beijing 100084, China
}

Correspondence should be addressed to Xiao-Gang Wen; wen@dao.mit.edu

Received 2 October 2012; Accepted 19 November 2012

Academic Editors: H. Eisaki, C. Janowitz, C. Trallero-Giner, and T. Yanagisawa

Copyright (C) 2013 Xiao-Gang Wen. This is an open access article distributed under the Creative Commons Attribution License, which permits unrestricted use, distribution, and reproduction in any medium, provided the original work is properly cited.

\begin{abstract}
We review the progress in the last 20-30 years, during which we discovered that there are many new phases of matter that are beyond the traditional Landau symmetry breaking theory. We discuss new "topological" phenomena, such as topological degeneracy that reveals the existence of those new phases-topologically ordered phases. Just like zero viscosity defines the superfluid order, the new "topological" phenomena define the topological order at macroscopic level. More recently, we found that at the microscopical level, topological order is due to long-range quantum entanglements. Long-range quantum entanglements lead to many amazing emergent phenomena, such as fractional charges and fractional statistics. Long-range quantum entanglements can even provide a unified origin of light and electrons; light is a fluctuation of long-range entanglements, and electrons are defects in long-range entanglements.
\end{abstract}

\section{Introduction}

1.1. Phases of Matter and Landau Symmetry-Breaking Theory. Although all matter is formed by only three kinds of particles: electrons, protons, and neutrons, matter can have many different properties and appear in many different forms, such as solid, liquid, conductor, insulator, superfluid, and magnet. According to the principle of emergence in condensed matter physics, the rich properties of materials originate from the rich ways in which the particles are organized in the materials. Those different organizations of the particles are formally called the orders in the materials.

For example, particles have a random distribution in a liquid (see Figure 1(a)), so a liquid remains the same as we displace it by an arbitrary distance. We say that a liquid has a "continuous translation symmetry." After a phase transition, a liquid can turn into a crystal. In a crystal, particles organize into a regular array (a lattice) (see Figure 1(b)). A lattice remains unchanged only when we displace it by a particular set of distances (integer times of lattice constant), so a crystal has only "discrete translation symmetry." The phase transition between a liquid and a crystal is a transition that reduces the continuous translation symmetry of the liquid to the discrete symmetry of the crystal. Such a change in symmetry is called "spontaneous symmetry breaking." We note that the equation of motions that governs the dynamics of the particles respects the continuous translation symmetry for both cases of liquid and crystal. However, in the case of crystal, the stronger interaction makes the particles to prefer being separated by a fixed distance and a fixed angle. This makes particles to break the continuous translation symmetry down to discrete translation symmetry "spontaneously" in order to choose a low-energy configuration (see Figure 2). Therefore, the essence of the difference between liquids and crystals is that the organizations of particles have different symmetries in the two phases.

Liquid and crystal are just two examples. In fact, particles can organize in many ways which lead to many different orders and many different types of materials. Landau symmetry-breaking theory [1-3] provides a general and a systematic understanding of these different orders. It points out that different orders really correspond to different symmetries in the organizations of the constituent particles. As a material changes from one order to another order 


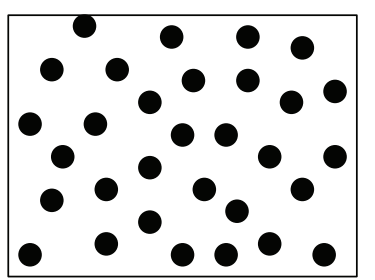

(a)

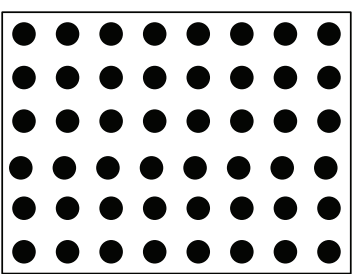

(b)

Figure 1: (a) Particles in liquids do not have fixed relative positions. They fluctuate freely and have a random but uniform distribution. (b) Particles in solids form a fixed regular lattice.

(i.e., as the material undergoes a phase transition), what happens is that the symmetry of the organization of the particles changes. Landau symmetry-breaking theory is a very successful theory. For a long time, physicists believed that Landau symmetry-breaking theory describes all possible orders in materials and all possible (continuous) phase transitions.

1.2. Quantum Phases of Matter. Quantum phases of matter are phases of matter at zero temperature. So quantum phases correspond to the ground states of the quantum Hamiltonians that govern the systems. In this paper, we will mainly discuss those quantum phases of matter. Crystal, conductor, insulator, superfluid, and magnets can exist at zero temperature and are examples of quantum phases of matter.

Again, physicists used to believe that Landau symmetrybreaking theory also describes all possible quantum phases of matter and all possible (continuous) quantum phase transitions. (Quantum phase transitions are zero temperature phase transitions.) For example, the superfluid is described by a $U(1)$ symmetry breaking.

It is interesting to compare a finite-temperature phase, liquid, with a zero-temperature phase, superfluid. A liquid is described as a random probability distributions of particles (such as atoms), while a superfluid is described by a quantum wave function which is the superposition of a set of random particle configurations:

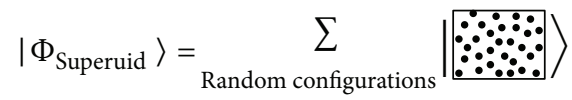

The superpositions of many different particle positions are called quantum fluctuations in particle positions.

Since Landau symmetry-breaking theory suggests that all quantum phases are described by symmetry breaking, thus we can use group theory to classify all those symmetrybreaking phases. All symmetry-breaking quantum phases are classified by a pair of mathematical objects $\left(G_{H}, G_{\Phi}\right)$, where $G_{H}$ is the symmetry group of the Hamiltonian and $G_{\Phi}$ is the symmetry group of the ground state. For example, a boson superfluid is labeled by $(U(1),\{1\})$, where $U(1)$ is the symmetry group of the boson Hamiltonian which conserves the boson number and $\{1\}$ is the trivial group that contains only identity.

\section{Topological Order}

2.1. The Discovery of Topological Order. However, in late 1980s, it became clear that Landau symmetry-breaking theory did not describe all possible phases. In an attempt to explain high-temperature superconductivity, the chiral spin state was introduced [4,5]. At first, physicists still wanted to use Landau symmetry-breaking theory to describe the chiral spin state. They identified the chiral spin state as a state that breaks the time reversal and parity symmetries, but not the spin rotation symmetry [5]. This should be the end of story according to Landau symmetry-breaking description of orders.

But, it was quickly realized that there are many different chiral spin states that have exactly the same symmetry [6]. So symmetry alone was not enough to characterize and distinguish different chiral spin states. This means that the chiral spin states must contain a new kind of order that is beyond the usual symmetry description. The proposed new kind of order was named "topological order" [7]. (The name "topological order" was motivated by the low-energy effective theory of the chiral spin states which is a Chern-Simons theory [5] - a topological quantum field theory (TQFT) [8].) New quantum numbers (or new topological probes), such as ground-state degeneracy $[6,9]$ and the non-Abelian geometric phase of degenerate ground states $[7,10]$, were introduced to characterize/define the different topological orders in chiral spin states.

But experiments soon indicated that chiral spin states do not describe high-temperature superconductors, and the theory of topological order became a theory with no experimental realization. However, the similarity [4] between chiral spin states and fractional quantum Hall $(\mathrm{FQH})$ states $[11,12]$ allows one to use the theory of topological order to describe different FQH states.

$\mathrm{FQH}$ states are gapped ground states of $2 \mathrm{D}$ electrons under strong magnetic field. $\mathrm{FQH}$ states have a property that a current density will induce an electric field in the transverse direction: $E_{y}=R_{H} j_{x}$ (see Figure 3). It is an amazing discovery that the Hall coefficient $R_{H}$ of a FQH state is precisely quantized as a rational number $p / q$ if we measure the Hall coefficient $R_{H}$ in unit of $\left(h / e^{2}\right): R_{H}=(p / q)\left(h / e^{2}\right)$ (see Figure 3) [11]. Different quantized $R_{H}$ correspond to different FQH states. Just like the chiral spin states, different $\mathrm{FQH}$ states all have the same symmetry and cannot be distinguished by symmetry breaking. So there is no way to use 


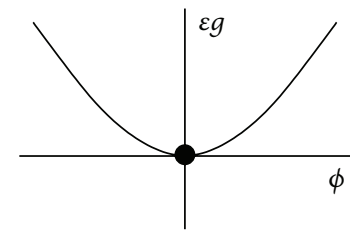

(a)
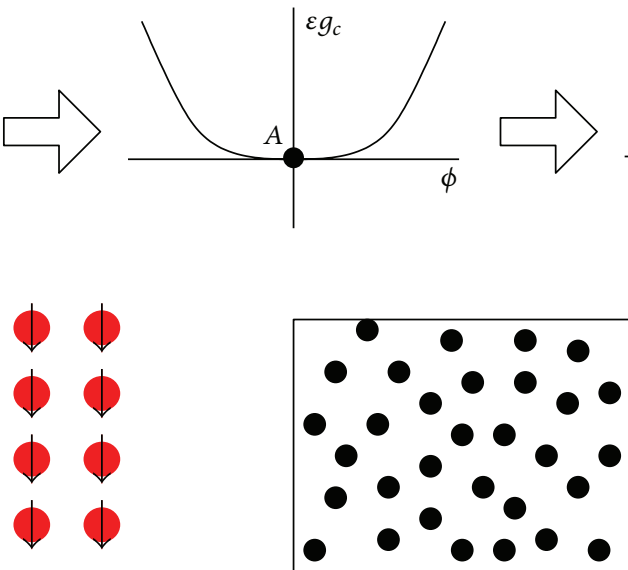

(a)

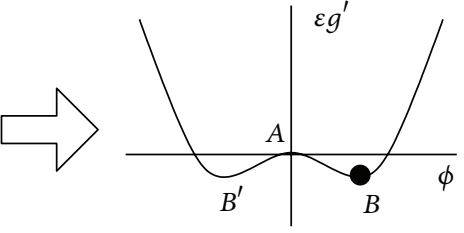

(b)

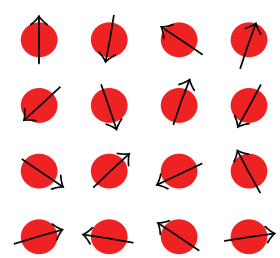

(a)

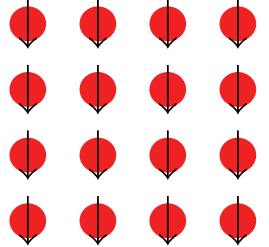

(b)

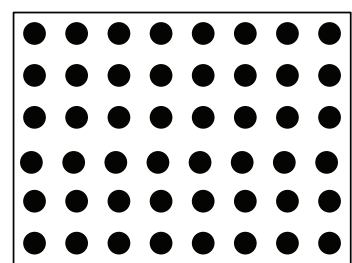

(b)

Figure 2: (a) Disordered states that do not break the symmetry. (b) Ordered states that spontaneously break the symmetry. The energy function $\varepsilon_{g}(\phi)$ has a symmetry $\phi \rightarrow-\phi: \varepsilon_{g}(\phi)=\varepsilon_{g}(-\phi)$. However, as we change the parameter $g$, the minimal energy state (the ground state) may respect the symmetry (a), or may not respect the symmetry (b). This is the essence of spontaneous symmetry breaking.
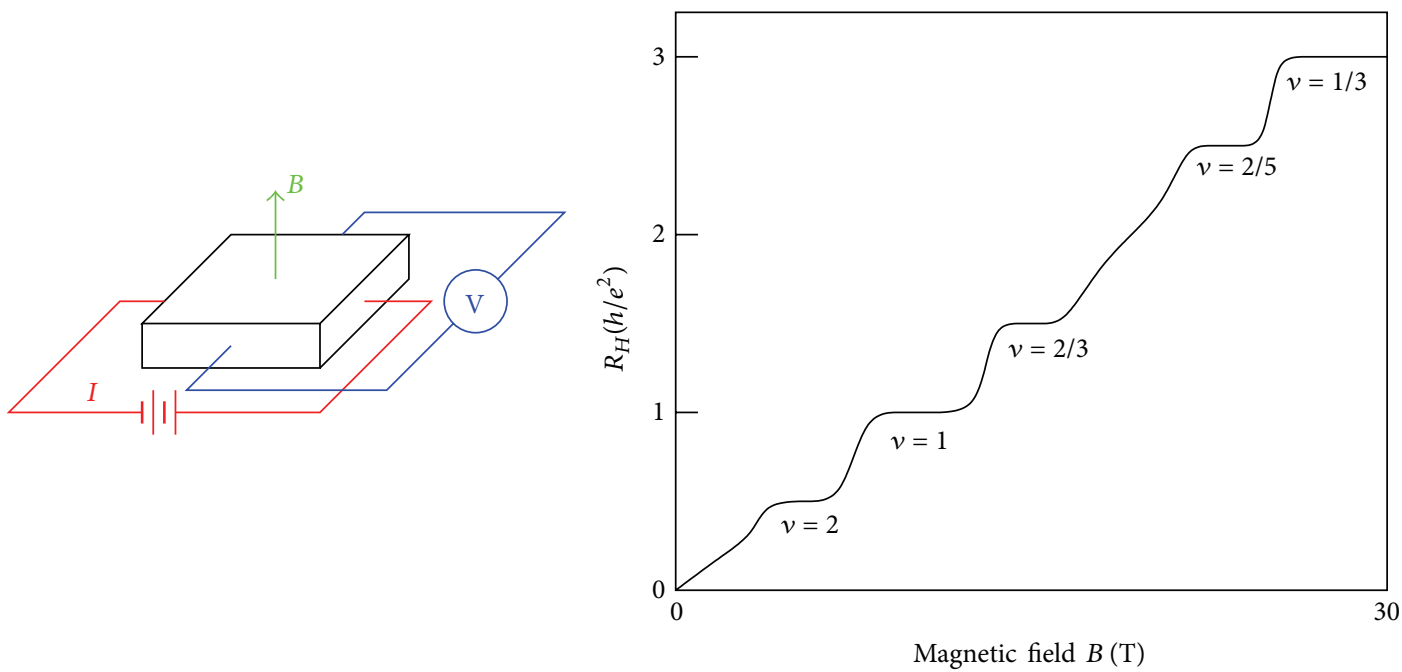

FIGURE 3: 2D electrons in strong magnetic field may form FQH states. Each FQH state has a quantized Hall coefficient $R_{H}$.

different symmetry breaking to describe different FQH states, and FQH states must contain new orders. One finds that the new orders in quantum Hall states can indeed be described by topological orders [9]. So, the topological order does have experimental realizations.

We would like to point out that before the topologicalorder understanding of FQH states, people have tried to use the notions of off-diagonal long-range order and order parameter from Ginzburg-Landau theory to describe FQH states [13-16]. Such an effort leads to a Ginzburg-Landau Chern-Simons effective theory for FQH states $[15,16]$. At the same time, it was also realized that the order parameter in the Ginzburg-Landau Chern-Simons is not gauge invariant and is not physical. This is consistent with the topological order understanding of FQH states which suggests that FQH has no off-diagonal long-range order and cannot be described by local order parameters. So, we can use effective theories without order parameters to describe FQH states, and such effective theories are pure Chern-Simons effective theories [9, 17-21]. The pure Chern-Simons effective theories lead to a K-matrix classification [20] of all Abelian topologically ordered states (which include all Abelian FQH states).

FQH states were discovered in 1982 [11] before the introduction of the concept of topological order. But FQH states are not the first experimentally discovered topologically ordered states. The real-life superconductors, having a $Z_{2}$ topological order [22-24], were the first experimentally discovered topologically ordered states. (Note that real-life superconductivity can be described by the Ginzburg-Landau theory with a dynamical $U(1)$ gauge field, which becomes a $Z_{2}$ gauge theory at low energies, and a $Z_{2}$ gauge theory is an effective theory of $Z_{2}$ topological order. In many textbook, superconductivity is described by the GinzburgLandau theory without the dynamical $U(1)$ gauge field, which 


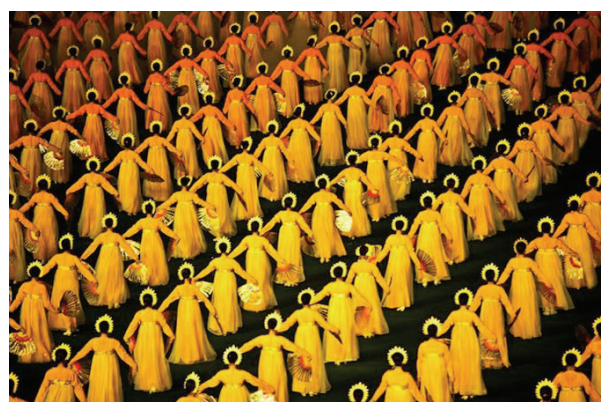

Ferromagnet

(a)

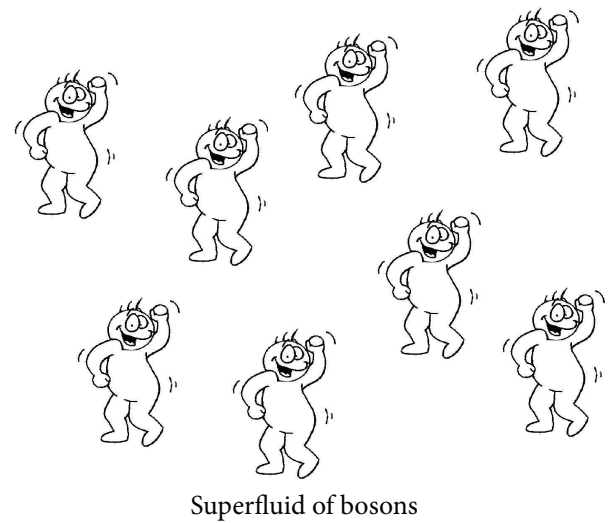

(c)

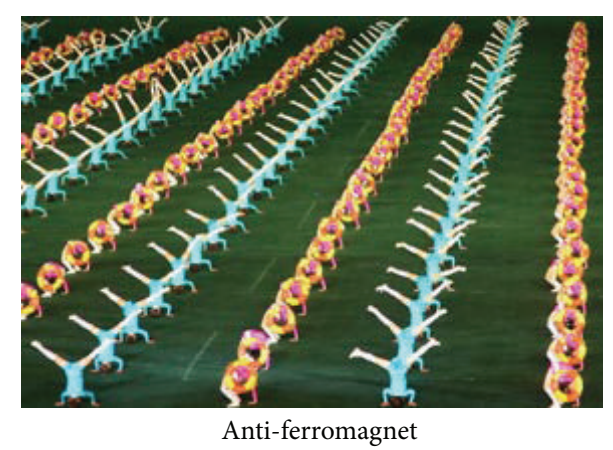

(b)

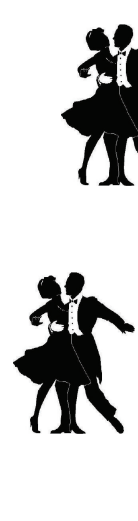

Superfluid of fermions

(d)

FIGURE 4: The dancing patterns for the symmetry-breaking orders.

fails to describe the real-life superconductors with dynamical electromagnetic interaction. Such a textbook superconductivity is described by a $U(1)$ symmetry breaking.) (Ironically, the Ginzburg-Landau symmetry-breaking theory was developed to describe superconductors, though the reallife superconductors are not symmetry-breaking states, but topologically ordered states.)

2.2. Intuitive Pictures of Topological Order. Topological order is a very new concept that describes quantum entanglements in many body systems. Such a concept is very remote from our daily experiences, and it is hard to have an intuition about it. So, before we define topological order in general terms (which can be abstract), let us first introduce and explain the concept through some intuitive pictures.

We can use dancing to gain an intuitive picture of topological order. But before we do that, let us use dancing picture to describe the old symmetry-breaking orders (see Figure 4). In the symmetry-breaking orders, every particle/spin (or every pair of particles/spins) dances by itself, and they all dance in the same way. (The "same way" of dancing represents a long-range order.) For example, in a ferromagnet, every electron has a fixed position and the same spin direction. We can describe an antiferromagnet by saying every pair of electrons has a fixed position, and the two electrons in a pair have opposite spin directions. In a boson superfluid, each boson is moving around by itself and doing the same dance, while in a fermion superfluid, fermions dance around in pairs, and each pair is doing the same dance.

We can also understand topological orders through such dancing pictures (see Figure 5). Unlike fermion superfluid where fermions dance in pairs, a topological order is described by a global dance, where every particle (or spin) is dancing with every other particle (or spin) in a very organized way. (a) All spins/particles dance following a set of local dancing "rules" trying to lower the energy of a local Hamiltonian. (b) If all the spins/particles follow the local dancing "rules," then they will form a global dancing pattern, which corresponds to the topological order. (c) Such a global pattern of collective dancing is a pattern of quantum fluctuation which corresponds to a pattern of long-range entanglements.

For example, in FQH liquid, the electrons dance following the following local dancing rules.

(a) Electron always dances anticlockwise which implies that the electron wave function only depends on the electron coordinates $(x, y)$ via $z=x+i y$.

(b) Each electron always takes exact three steps to dance around any other electron, which implies that the phase of the wave function changes by $6 \pi$ as we move an electron around any other electron.

The above two local dancing rules fix a global dance pattern which corresponds to the Laughlin wave function [12] 


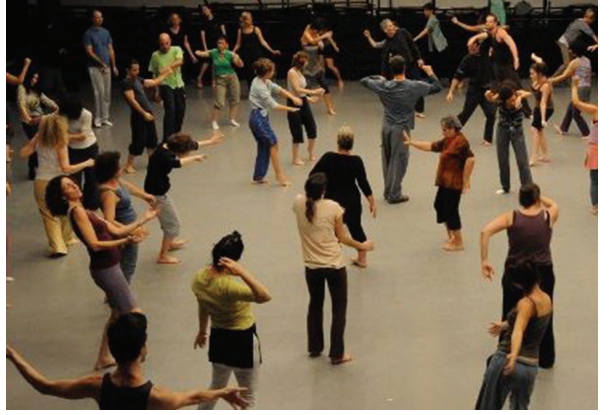

$\mathrm{FQH}$ state

(a)

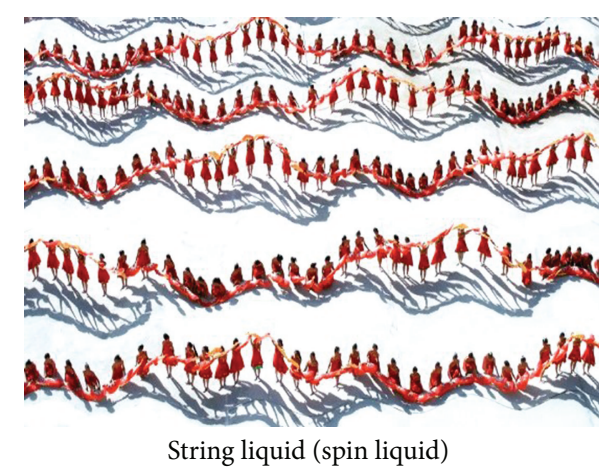

(b)

FIGURE 5: The dancing patterns for the topological orders.

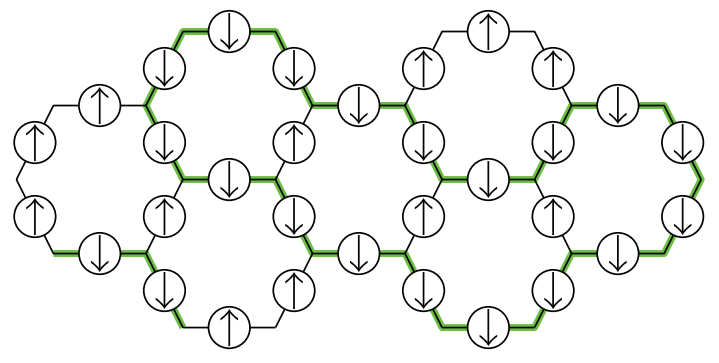

Figure 6: The strings in a spin-1/2 model. In the background of upspins, the down-spins form closed strings.

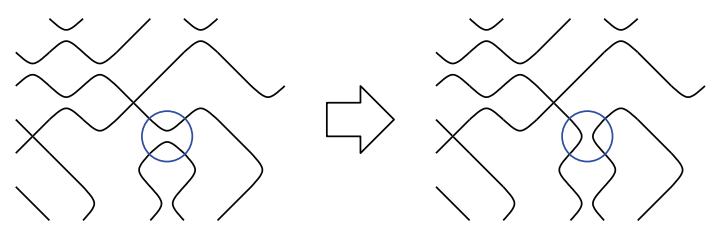

FIgURE 7: In the string liquid, strings can move freely, including reconnecting the strings.

$\Phi_{\mathrm{FQH}}=\prod\left(z_{i}-z_{j}\right)^{3}$. Such a collective dancing gives rise to the topological order (or long-range entanglements) in the FQH state.

In addition to FQH states, some spin liquids also contain topological orders [5, 23, 25-27]. (Spin liquids refer to ground states of quantum spin systems that do not break the spin rotation and the translation symmetries in the spin Hamiltonians.) In those spin liquids, the spins "dance" following the following local dancing rules:

(a) down-spins form closed strings with no ends in the background of up-spins (see Figure 6);

(b) strings can otherwise move freely, including reconnecting freely (see Figure 7).

The global dance formed by the spins following the above dancing rules gives us a quantum spin liquid which is a superposition of all closed-string configurations [28]: $\left.\left|\Phi_{\text {string }}\right\rangle=\sum_{\text {all string pattern }}|\vec{i} \widetilde{\sim}\rangle_{i}\right\rangle$. Such a state is called a string or string-net condensed state [29]. The collective dancing gives rise to a nontrivial topological order and a pattern of long-range entanglements in the spin-liquid state.

\section{What Is the Significance of Topological Order?}

The above descriptions of topological order are intuitive and not concrete. It is not clear if the topological order (the global dancing pattern or the long-range entanglement) has any experimental significance. In order for the topological order to be a useful concept, it must have new experimental properties that are different from any symmetry-breaking states. Those new experimental properties should indicate the nontrivialness of the topological order. In fact, the concept of topological order should be defined by the collection of those new experimental properties.

Indeed, topological order does have new characteristic properties. Those properties of topological orders reflect the significance of topological order.

(1) Topological orders produce new kind of waves (i.e., the collective excitations above the topologically ordered ground states) [30-38]. The new kind of waves can be probed/studied in practical experiments, such as neutron scattering experiments [34].

(2) The finite-energy defects of topological order (i.e., the quasiparticles) can carry fractional statistics [39, 40] (including non-Abelian statistics [41, 42]) and fractional charges $[12,43]$ (if there is a symmetry). Such a property allows us to use topologically ordered states as a medium for topological quantum memory [44] and topological quantum computations [28].

(3) Some topological orders have topologically protected gapless boundary excitations [45-47]. Such gapless boundary excitations are topologically protected, which lead to perfect conducting boundary channels even with magnetic impurities [48]. This property may lead to device applications.

In the following, we will study some examples of topological orders and reveal their amazing topological properties. 


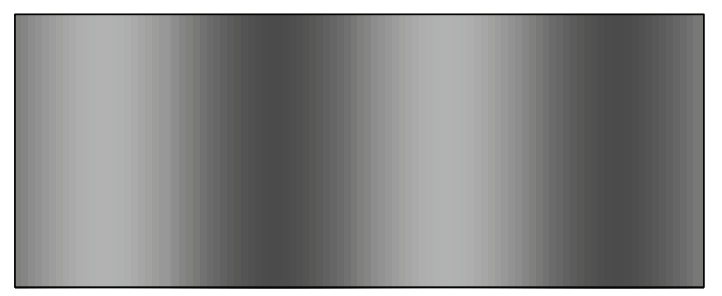

FIGURE 8: Liquids only have a compression wave-a wave of density fluctuations.

\section{Examples of Topological Order: A Quantum Liquid of Oriented Strings and a Unification of Gauge Interactions and Fermi Statistics}

Our first example is a quantum liquid of oriented strings. We will discuss its new topological properties (1) and (2). We find that the new kind of waves and the emergent statistics are so profound, that they may change our view of universe. Let us start by explaining a basic notion- "principle of emergence".

4.1. Principle of Emergence. Typically, one thinks that the properties of a material should be determined by the components that form the material. However, this simple intuition is incorrect, since all the materials are made of same components: electrons, protons, and neutrons. So, we cannot use the richness of the components to understand the richness of the materials. In fact, the various properties of different materials originate from various ways in which the particles are organized. Different orders (the organizations of particles) give rise to different physical properties of a material. It is the richness of the orders that gives rise to the richness of material world.

Let us use the origin of mechanical properties and the origin of waves to explain, in a more concrete way, how orders determine the physics properties of a material. We know that a deformation in a material can propagate just like the ripple on the surface of water. The propagating deformation corresponds to a wave traveling through the material. Since liquids can resist only compression deformation, so liquids can only support a single kind of wave-compression wave (see Figure 8). (Compression wave is also called longitudinal wave.) Mathematically, the motion of the compression wave is governed by the Euler equation

$$
\frac{\partial^{2} \rho}{\partial t^{2}}-v^{2} \frac{\partial^{2} \rho}{\partial x^{2}}=0
$$

where $\rho$ is the density of the liquid.

Solid can resist both compression and shear deformations. As a result, solids can support both compression wave and transverse wave. The transverse wave corresponds to the propagation of shear deformations. In fact, there are two transverse waves corresponding to two directions of shear deformations. The propagation of the compression wave and

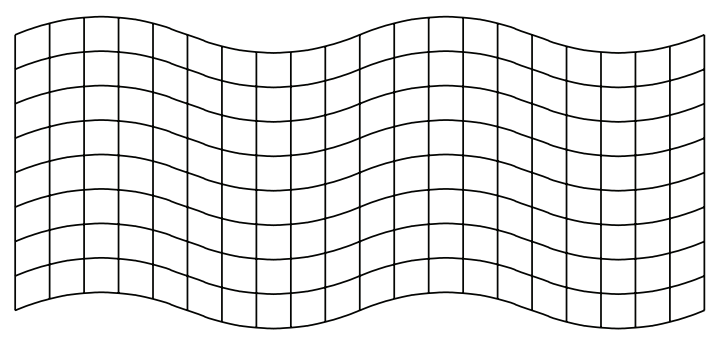

FIGURE 9: Drawing a grid on a sold helps us to see the deformation of the solid. The vector $u^{i}$ in (3) is the displacement of a vertex in the grid. In addition to the compression wave (i.e., the density wave), a solid also supports transverse wave (wave of shear deformation) as shown in the above figure.

the two transverse waves in solids are described by the elasticity equation

$$
\frac{\partial^{2} u^{i}}{\partial t^{2}}-T_{j}^{i k l} \frac{\partial^{2} u^{j}}{\partial x^{k} \partial x^{l}}=0
$$

where the vector field $u^{i}(\mathbf{x}, t)$ describes the local displacement of the solid (see Figure 9).

We would like to point out that the elasticity equation and the Euler equations not only describe the propagation of waves, but also they actually describe all small deformations in solids and liquids. Thus, the two equations represent a complete mathematical description of the mechanical properties of solids and liquids.

But why do solids and liquids behave so differently? What makes a solid to have a shape and a liquid to have no shape? What are the origins of elasticity equation and Euler equations? The answer to those questions has to wait until the discovery of atoms in 19th century. Since then, we realized that both solids and liquids are formed by collections of atoms. The main difference between the solids and liquids is that the atoms are organized very differently. In liquids, the positions of atoms fluctuate randomly (see Figure 1(a)), while in solids, atoms organize into a regular fixed array (see Figure 1(b)). (The solids here should be more accurately referred as crystals.) It is the different organizations of atoms that lead to the different mechanical properties of liquids and solids. In other words, it is the different organizations of atoms that make liquids to be able to flow freely and solids to be able to retain their shape.

How can different organizations of atoms affect mechanical properties of materials? In solids, both the compression deformation (see Figure 10(a)) and the shear deformation (see Figure 10(b)) lead to real physical changes of the atomic configurations. Such changes cost energies. As a result, solids can resist both kinds of deformations and can retain their shapes. This is why we have both the compression wave and the transverse wave in solids.

In contrast, a shear deformation of atoms in liquids does not result in a new configuration since the atoms still have uniformly random positions. So, the shear deformation is a do-nothing operation for liquids. Only the compression deformation which changes the density of the atoms results in a new atomic configuration and costs energies (see Figure 11). 


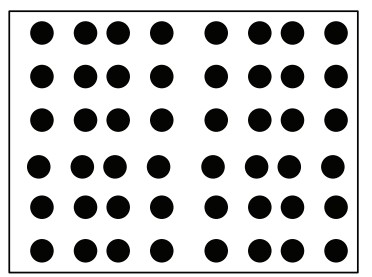

(a)

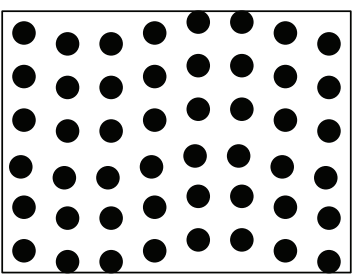

(b)

FIgURE 10: The atomic picture of (a) the compression wave and (b) the transverse wave in a crystal.

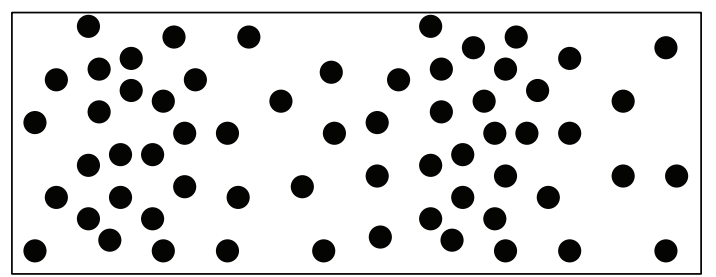

FIGURE 11: The atomic picture of the compression wave in liquids.

As a result, liquids can only resist compression and have only compression wave. Since shear deformations do not cost any energy for liquids, liquids can flow freely.

We see that the properties of the propagating wave are entirely determined by how the atoms are organized in the materials. Different organizations lead to different kinds of waves and different kinds of mechanical laws. Such a point of view of different kinds of waves/laws originated from different organizations of particles is a central theme in condensed matter physics. This point of view is called the principle of emergence.

4.2. String-Net Liquid Unifies Light and Electrons. The elasticity equation and the Euler equation are two very important equations. They lay the foundation of many branches of science, such as mechanical engineering and aerodynamic engineering. But, we have a more important equation, Maxwell equation, that describes light waves in vacuum. When Maxwell equation was first introduced, people firmly believed that any wave must correspond to motion of something. So, people want to find out what is the origin of the Maxwell equation? The motion of what gives rise to electromagnetic wave?

First, one may wonder, can Maxwell equation comes from a certain symmetry-breaking order? Based on Landau symmetry-breaking theory, the different symmetry-breaking orders can indeed lead to different waves satisfying different wave equations. So, maybe a certain symmetry-breaking order can give rise to a wave that satisfies Maxwell equation. But people have been searching for ether-a medium that supports light wave-for over 100 years and could not find any symmetry-breaking states that can give rise to waves satisfying the Maxwell equation. This is one of the reasons why people give up the idea of ether as the origin of light and Maxwell equation.

However, the discovery of topological order [6, 7] suggests that Landau symmetry-breaking theory does not describe all possible organizations of bosons/spins. This gives us a new hope; Maxwell equation may arise from a new kind of organizations of bosons/spins that have nontrivial topological orders.

In addition to the Maxwell equation, there is an even stranger equation, Dirac equation, that describes wave of electrons (and other fermions). Electrons have Fermi statistics. They are fundamentally different from the quanta of other familiar waves, such as photons and phonons, since those quanta all have Bose statistics. To describe the electron wave, the amplitude of the wave must be anticommuting Grassmann numbers, so that the wave quanta will have Fermi statistics. Since electrons are so strange, few people regard electrons and the electron waves as collective motions of something. People accept without questioning that electrons are fundamental particles, one of the building blocks of all that exist.

However, from a condensed matter physics point of view, all low-energy excitations are collective motion of something. If we try to regard photons as collective modes, why cannot we regard electrons as collective modes as well? So, maybe Dirac equation and the associated fermions can also arise from a new kind of organizations of bosons/spins that have nontrivial topological orders.

A recent study provides a positive answer to the above questions [29, 36, 37]. We find that if bosons/spins form large oriented strings and if those strings form a quantum liquid state, then the collective motion of such organized bosons/spins will correspond to waves described by Maxwell equation and Dirac equation. The strings in the string liquid are free to join and cross each other. As a result, the strings look more like a network (see Figure 12). For this reason, the string liquid is actually a liquid of string-nets, which is called string-net condensed state.

But why does the waving of strings produce waves described by the Maxwell equation? We know that the particles in a liquid have a random but uniform distribution. A deformation of such a distribution corresponds to a density fluctuation, which can be described by a scaler field $\rho(\mathbf{x}, t)$. Thus, the waves in a liquid are described by the scaler field $\rho(\mathbf{x}, t)$, which satisfy the Euler equation (2). Similarly, the strings in a string-net liquid also have a random but uniform distribution (see Figure 13). A deformation of string-net liquid corresponds to a change of the density of the strings (see Figure 14). However, since strings have an orientation, the "density" fluctuations are described by a vector field $\mathbf{E}(\mathbf{x}, t)$, which indicates that there are more strings in the $\mathbf{E}$ 


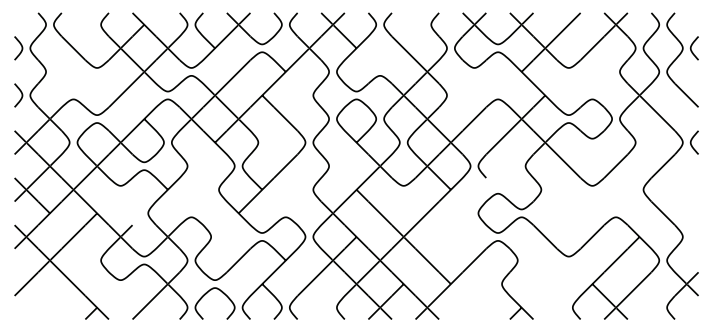

FIGURE 12: A quantum ether: the fluctuation of oriented strings gives rise to electromagnetic waves (or light). The ends of strings give rise to electrons. Note that oriented strings have directions which should be described by curves with arrow. For ease of drawing, the arrows on the curves are omitted in the above plot.

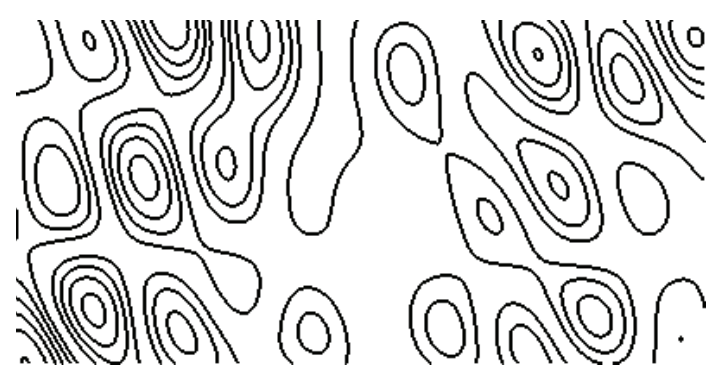

FIGURE 13: The fluctuating strings in a string liquid.

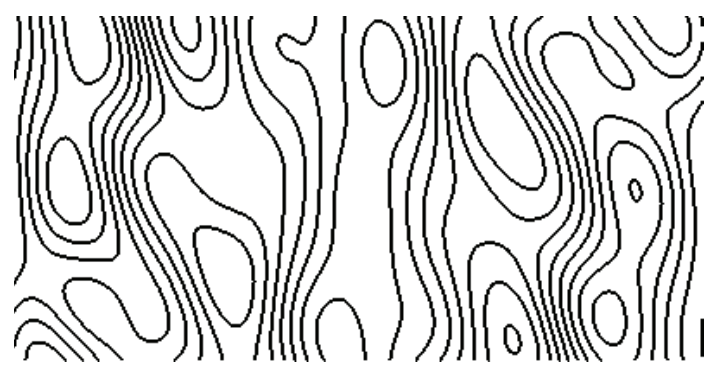

FIGURE 14: A "density" wave of oriented strings in a string liquid. The wave propagates in $x$-direction. The "density" vector $\mathbf{E}$ points in $y$-direction. For ease of drawing, the arrows on the oriented strings are omitted in the above plot.

direction on average. The oriented strings can be regarded as flux lines. The vector field $\mathbf{E}(\mathbf{x}, t)$ describes the smeared average flux. Since strings are continuous (i.e., they cannot end), the flux is conserved: $\partial \cdot \mathbf{E}(\mathbf{x}, t)=0$. The vector density $\mathbf{E}(\mathbf{x}, t)$ of strings cannot change in the direction along the strings (i.e., along the $\mathbf{E}(\mathbf{x}, t)$ direction). $\mathbf{E}(\mathbf{x}, t)$ can change only in the direction perpendicular to $\mathbf{E}(\mathbf{x}, t)$. Since the direction of the propagation is the same as the direction in which $\mathbf{E}(\mathbf{x}, t)$ varies, thus the waves described by $\mathbf{E}(\mathbf{x}, t)$ must be transverse waves; $\mathbf{E}(\mathbf{x}, t)$ is always perpendicular to the direction of the propagation. Therefore, the waves in the string liquid have a very special property; the waves have only transverse modes and no longitudinal mode. This is exactly the property of the light waves described by the Maxwell equation. We see that "density" fluctuations of strings (which are described by a transverse vector field) naturally give rise to the light (or electromagnetic) waves and the Maxwell equation [32-37].

It is interesting to compare solid, liquid, and string-net liquid. We know that the particles in a solid organized into a regular lattice pattern. The waving of such organized particles produces a compression wave and two transverse waves. The particles in a liquid have a more random organization. As a result, the waves in liquids lost two transverse modes and contained only a single compression mode. The particles in a string-net liquid also have a random organization, but in a different way. The particles first form string-nets, and stringnets then form a random liquid state. Due to this different kind of randomness, the waves in string-net condensed state lost the compression mode and contained two transverse modes. Such a wave (having only two transverse modes) is exactly the electromagnetic wave.

To understand how electrons appear from string-nets, we would like to point out that if we only want photons and no other particles, the strings must be closed strings with no ends. The fluctuations of closed strings produce only photons. If strings have open ends, those open ends can move around and just behave like independent particles. Those particles are not photons. In fact, the ends of strings are nothing but electrons.

How do we know that ends of strings behave like electrons? First, since the waving of string-nets is an electromagnetic wave, a deformation of string-nets corresponds to an electromagnetic field. So, we can study how an end of a string interacts with a deformation of string-nets. We find that such an interaction is just like the interaction between a charged electron and an electromagnetic field. Also electrons have a subtle but very important property-Fermi statistics, which is a property that exists only in quantum theory. Amazingly, the ends of strings can reproduce this subtle quantum property of Fermi statistics $[29,49]$. Actually, string-net liquids explain why Fermi statistics should exist.

We see that string-nets naturally explain both light and electrons (gauge interactions and Fermi statistics). In other words, string-net theory provides a way to unify light and electrons $[36,37]$. So, the fact that our vacuum contains both light and electrons may not be a mere accident. It may actually suggest that the vacuum is indeed a string-net liquid.

4.3. More General String-Net Liquid and Emergence of NonAbelian Gauge Theory. Here, we would like to point out that there are many different kinds of string-net liquids. The strings in different liquids may have different numbers of types. The strings may also join in different ways. For a general string-net liquid, the waving of the strings may not correspond to light, and the ends of strings may not be electrons. Only one kind of string-net liquids gives rise to light and electrons. On the other hand, the fact that there are many different kinds of string-net liquids allows us to explain more than just light and electrons. We can design a particular type of string-net liquids which not only gives rise to electrons and photons, but also gives rise to quarks and gluons $[29,33]$. The waving of such type of stringnets corresponds to photons (light) and gluons. The ends of different types of strings correspond to electrons and quarks. 
It would be interesting to see if it is possible to design a stringnet liquid that produces all elementary particles! If this is possible, the ether formed by such string-nets can provide an origin of all elementary particles. (So far we can use stringnet to produce almost all elementary particles, expect for the graviton that is responsible for the gravity. Also, we are unable to produce the chiral coupling between the $S U(2)$ gauge boson and the fermions within the string-net picture.)

We like to stress that the string-nets are formed by qubits. So, in the string-net picture, both the Maxwell equation and Dirac equation emerge from local qubit model, as long as the qubits are from a long-range entangled state (i.e., a string-net liquid). In other words, light and electrons are unified by the long-range entanglements of qubits!

The electric field and the magnetic field in the Maxwell equation are called gauge fields. The fields in the Dirac equation are Grassmann-number valued field. (Grassmann numbers are anticommuting numbers. For a long time, we thought that we have to use gauge fields to describe light waves that have only two transverse modes, and we thought that we have to use Grassmann-number valued fields to describe electrons and quarks that have Fermi statistics. So, gauge fields and Grassmann-number valued fields became the fundamental build blocks of quantum field theory that describes our world. The string-net liquids demonstrate that we do not have to introduce gauge fields and Grassmannnumber valued fields to describe photons, gluons, electrons, and quarks. It demonstrates how gauge fields and Grassmann fields emerge from local qubit models that contain only complex scaler fields at the cut-off scale.

Our attempt to understand light has a long and evolving history. We first thought light to be a beam of particles. After Maxwell, we understand light as electromagnetic waves. After Einstein's theory of general relativity, where gravity is viewed as curvature in space-time, Weyl and others try to view electromagnetic field as curvatures in the "unit system" that we used to measure complex phases. It leads to the notion of gauge theory. The general relativity and the gauge theory are two corner stones of modern physics. They provide a unified understanding of all four interactions in terms of a beautiful mathematical framework; all interactions can be understood geometrically as curvatures in space-time and in "unit systems" (or more precisely, as curvatures in the tangent bundle and other vector bundles in space-time).

Later, people in high-energy physics and in condensed matter physics have found another way in which gauge field can emerge [50-53]; one first cut a particle (such as an electron) into two partons by writing the field of the particle as the product of the two fields of the two partons. Then one introduces a gauge field to glue the two partons back to the original particle. Such a "glue picture" of gauge fields (instead of the fiber bundle picture of gauge fields) allows us to understand the emergence of gauge fields in models that originally contain no gauge field at the cut-off scale.

A string picture represents the third way to understand gauge theory. String operators appear in the Wilson-loop characterization [54] of gauge theory. The Hamiltonian and the duality description of lattice gauge theory also reveal string structures [55-58]. Lattice gauge theories are not local bosonic models, and the strings are unbreakable in lattice gauge theories. String-net theory points out that even breakable strings can give rise to gauge fields [59]. So, we do not really need strings. Qubits themselves are capable of generating gauge fields and the associated Maxwell equation. This phenomenon was discovered in several qubit models $[30,35,52,60,61]$ before realizing their connection to the string-net liquids [32]. Since gauge field can emerge from local qubit models, the string picture evolves into the entanglement picture - the fourth way to understand gauge field; gauge fields are fluctuations of long-range entanglements. I feel that the entanglement picture captures the essence of gauge theory. Despite the beauty of the geometric picture, the essence of gauge theory is not the curved fiber bundles. In fact, we can view gauge theory as a theory for long-range entanglements, although the gauge theory is discovered long before the notion of long-range entanglements. The evolution of our understanding of light and gauge interaction: particle beam $\rightarrow$ wave $\rightarrow$ electromagnetic wave $\rightarrow$ curvature in fiber bundle $\rightarrow$ glue of partons $\rightarrow$ wave in string-net liquid $\rightarrow$ wave in long-range entanglements represents 200 year's effort of human race to unveil the mystery of universe.

Viewing gauge field (and the associated gauge bosons) as fluctuations of long-range entanglements has an added bonus; we can understand the origin of Fermi statistics in the same way; fermions emerge as defects of long-range entanglements, even though the original model is purely bosonic. Previously, there are two ways to obtain emergent fermions from purely bosonic model: by binding gauge charge and gauge flux in $(2+1) D[62,63]$ and by binding the charge and the monopole in a $U(1)$ gauge theory in $(3+1) D$ [64-68]. Using long-range entanglements and their stringnet realization, we can obtain the simultaneous emergence of both gauge bosons and fermions in any dimensions and for any gauge group [29, 33, 36, 49]. This result gives us hope that maybe every elementary particle is emergent and can be unified using local qubit models. Thus, long-range entanglements offer us a new option to view our world; maybe our vacuum is a long-range entangled state. It is the pattern of the long-range entanglement in the vacuum that determines the content and the structures of observed elementary particles. Such a picture has an experimental prediction that will be described in the next Section 4.4.

We like to point out that the string-net unification of gauge bosons and fermions is very different from the superstring theory for gauge bosons and fermions. In the stringnet theory, gauge bosons and fermions come from the qubits that form the space, and "string-net" is simply the name that describes how qubits are organized in the ground state. So string-net is not a thing, but a pattern of qubits. In the string-net theory, the gauge bosons are waves of collective fluctuations of the string-nets, and a fermion corresponds to one end of string. In contrast, gauge bosons and fermions come from strings in the superstring theory. Both gauge bosons and fermions correspond to small pieces of strings. Different vibrations of the small pieces of strings give rise to different kind of particles. The fermions in the superstring theory are put in by hand through the introduction of Grassmann fields. 
4.4. A Falsifiable Prediction of String-Net Unification of Gauge Interactions and Fermi Statistics. In the string-net unification of light and electrons $[36,37]$, we assume that the space is formed by a collection of qubits, and the qubits form a stringnet condensed state. Light waves are collective motions of the string-nets, and an electron corresponds to one end of string. Such a string-net unification of light and electrons has a falsifiable prediction; all fermionic excitations must carry some gauge charges $[29,49]$.

The $U(1) \times S U(2) \times S U(3)$ standard model for elementary particles contains fermionic excitations (such as neutrons and neutrinos) that do not carry any $U(1) \times S U(2) \times S U(3)$ gauge charge. So, according to the string-net theory, the $U(1) \times$ $S U(2) \times S U(3)$ standard model is incomplete. According to the string-net theory, our universe not only has $U(1) \times S U(2) \times$ $S U$ (3) gauge theory, but it must also contain other gauge theories. Those additional gauge theories may have a gauge group of $Z_{2}$ or other discrete groups. Those extra discrete gauge theories will lead to new cosmic strings which will appear in very early universe.

\section{Examples of Topological Order: Quantum Liquid of Unoriented Strings and Emergence of Statistics}

In the above, we discussed how light and electrons may emerge from a quantum liquid of orientable strings. We like to point out that quantum liquids of orientable strings are not the simplest topologically ordered state. Quantum liquids of unoriented strings are simpler topologically ordered states. In this section, we will discuss quantum liquids of unoriented strings and their topological properties. Using those simpler examples, we will discuss in detail how can ends of strings become fermions, or even anyons.

5.1. Quantum Liquids of Unoriented Strings and the Local "Dancing" Rules. The strings in quantum liquids of unoriented strings can be realized in a spin-1/2 model. We can view up-spins as background and lines of down-spins as the strings (see Figure 6). Clearly, such string is unoriented. The simplest topologically ordered state in such spin- $1 / 2$ system is given by the equal-weight superposition of all closed strings [28]: $\left|\Phi_{Z_{2}}\right\rangle=\sum_{\text {all closed strings }}\left|\widetilde{S}_{0}\right\rangle$. Such a wave function represents a global dancing pattern that corresponds to a nontrivial topological order.

As we have mentioned before, the global dancing pattern is determined by local dancing rules. What are those local rules that give rise to the global dancing pattern $\left|\Phi_{Z_{2}}\right\rangle=$ $\sum_{\text {all closed strings }}\left|\tilde{i} \tilde{S}_{0}\right\rangle$ ? The first rule is that, in the ground state, the down-spins are always connected with no open ends. To describe the second rule, we need to introduce the amplitudes of close strings in the ground state: $\Phi(\mathbb{i} ; \widetilde{Q})$. The ground state is given by

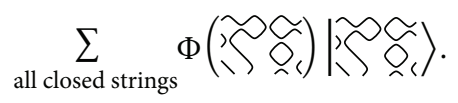

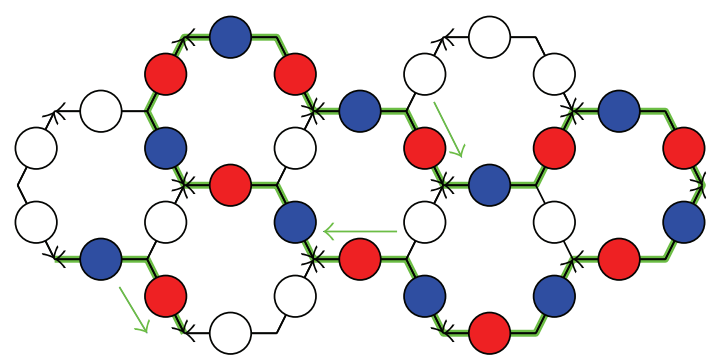

FIGURE 15: The orientable strings in a spin-1 model. In the background of $S_{z}=0$ spins (the white dots), the $S_{z}=1$ spins (the red dots) and the $S_{z}=-1$ spins (the blue dots) form closed strings.

Then, the second rule relates the amplitudes of close strings in the ground state as we change the strings locally:

$$
\Phi(\zeta)=\Phi(\square), \Phi(\square\langle)=\Phi(\square) .
$$

In other words, if we locally deform/reconnect the strings as in Figure 7, the amplitude (or the ground state wave function) does not change.

The first rule tells us that the amplitude of a string configuration only depends on the topology of the string configuration. Starting from a single loop, using the local deformation and the local reconnection in Figure 7, we can generate all closed string configurations with any number of loops. So all those closed string configurations have the same amplitude. Therefore, the local dancing rule fixes the wave function to be the equal-weight superposition of all closed strings: $\left|\Phi_{Z_{2}}\right\rangle=\sum_{\text {all closed strings }}\left|\widetilde{i} \tilde{\sigma}_{1}\right\rangle$. In other words, the local dancing rule fixes the global dancing pattern.

If we choose another local dancing rule, then we will get a different global dancing pattern that corresponds to a different topological order. One of the new choices is obtained by just modifying the sign in (5):

$$
\Phi(\zeta)=\Phi(\square), \Phi(\square\langle)=-\Phi(\square) .
$$

We note that each local reconnection operation changes the number of loops by 1 . Thus the new local dancing rules give rise to a wave function which has a form $\left|\Phi_{\text {Semi }}\right\rangle=$ $\sum_{\text {all closed strings }}(-)^{N_{\text {loops }}}\left|\vec{i} ; \tilde{O}_{1}\right\rangle$, where $N_{\text {loops }}$ is the number of loops. The wave function $\left|\Phi_{\text {Semi }}\right\rangle$ corresponds to a different global dance and a different topological order.

In the above, we constructed two quantum liquids of unoriented strings in a spin-1/2 model. Using a similar construction, we can also obtain a quantum liquid of orientable strings which gives rise to waves satisfying Maxwell equation as discussed before. To obtain quantum liquid of orientable strings, we need to start with a spin-1 model, where spins live on the links of honeycomb lattice (see Figure 15). Since the honeycomb lattice is bipartite, each link has an orientation from the A-sublattice to the B-sublattice (see Figure 15). The orientable strings are formed by alternating $S_{z}= \pm 1$ spins on the background of $S_{z}=0$ spins. The string orientation is given by the orientation of the links under the $S_{z}=1$ spins (see Figure 15). The superposition of the orientable strings gives rise to quantum liquid of orientable strings. 
5.2. Topological Properties of Quantum Liquids of Unoriented Strings. Why do the two wave functions of unoriented strings, $\left|\Phi_{Z_{2}}\right\rangle$ and $\left|\Phi_{\text {Semi }}\right\rangle$, have nontrivial topological orders? This is because the two wave functions give rise to nontrivial topological properties. The two wave functions correspond to different topological orders, since they give rise to different topological properties. In this section, we will discuss two topological properties: emergence of fractional statistics and topological degeneracy on compact spaces.

\subsubsection{Emergence of Fermi and Fractional Statistics. The two} topological states in two dimensions contain only closed strings, which represent the ground states. If the wave functions contain open strings (i.e., have nonzero amplitudes for open string states), then the ends of the open strings will correspond to point-like topological excitations above the ground states. Although an open string is an extended object, its middle part merges with the strings already in the ground states and is unobservable. Only its two ends carry energies and correspond to two point-like particles.

We note that such a point-like particle from an end of string cannot be created alone. Thus, an end of string corresponds to a topological point defect, which may carry fractional quantum numbers. This is because an open string as a whole always carries nonfractionalized quantum numbers. But an open string corresponds to two topological point defects from the two ends. So, we cannot say that each end of string carries nonfractionalized quantum numbers. Some times, they do carry fractionalized quantum numbers.

Let us first consider the defects in the $\left|\Phi_{Z_{2}}\right\rangle$ state. To understand the fractionalization, let us first consider the spin of such a defect to see if the spin is fractionalized or not $[69,70]$. An end of string can be represented by

$$
|i\rangle_{\mathrm{def}=}|i\rangle+|\dot{ }\rangle+|\dot{\gamma} 0\rangle+\cdots
$$

which is an equal-weight superposition of all string states obtained from the deformations and the reconnections of $\boldsymbol{i}$.

Under a $360^{\circ}$ rotation, the end of string is changed to (9) def, which is an equal-weight superposition of all string states obtained from the deformations and the reconnections of $\varphi$. Since |\rangle$_{\text {def }}$ and |\rangle$_{\text {def }}$ are always different, |\rangle$_{\text {def }}$ is not an eigenstate of $360^{\circ}$ rotation and does not carry a definite spin.

To construct the eigenstates of $360^{\circ}$ rotation, let us make a $360^{\circ}$ rotation to $\left|{ }^{9}\right\rangle_{\text {def. }}$. To do that, we first use the string reconnection move in Figure 7 to show that |\rangle$_{\text {def }}=\left|{ }^{\ominus}\right\rangle_{\text {def. }}$. A $360^{\circ}$ rotation on $\left|{ }^{\ominus}\right\rangle_{\text {def }}$ gives us |\rangle$_{\text {def. }}$.

We see that the $360^{\circ}$ rotation exchanges |\rangle$_{\text {def }}$ and $\left|{ }^{\beta}\right\rangle_{\text {def. }}$ Thus, the eigenstates of $360^{\circ}$ rotation are given by |\rangle$_{\text {def }}+|{ }\rangle_{\text {def }}$ with eigenvalue 1 and by |\rangle$_{\text {def }}-|\rangle_{\text {def }}$ with eigenvalue -1 . So, the particle $|i\rangle_{\text {def }}+|\rangle_{\text {def }}$ has a spin $0(\bmod 1)$, and the particle $|i\rangle_{\text {def }}-|\rangle_{\text {def }}$ has a spin $1 / 2(\bmod 1)$.

If one believes in the spin-statistics theorem, one may guess that the particle |\rangle$_{\text {def }}+|\rangle_{\text {def }}$ is a boson and the particle |\rangle$_{\text {def }}-|\rangle_{\text {def }}$ is a fermion. This guessing is indeed correct. From

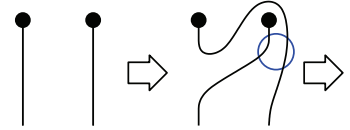

(a) (b)

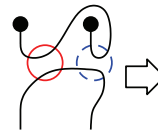

(c)

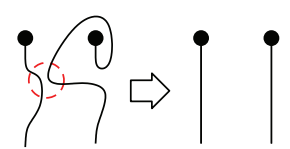

(d) (e)
FIGURE 16: Deformation of strings and two reconnection moves, plus an exchange of two ends of strings and a $360^{\circ}$ rotation of one of the ends of the string, change the configuration (a) back to itself. Note that from (a) to (b) we exchange the two ends of strings, and from (d) to (e) we rotate one of the ends of the string by $360^{\circ}$. The combination of those moves does not generate any phase.

Figure 16, we see that we can use deformation of strings and two reconnection moves to generate an exchange of two ends of strings and a $360^{\circ}$ rotation of one of the ends of the string. Such operations allow us to show that Figures 16(a) and 16(e) have the same amplitude, which means that an exchange of two ends of strings followed by a $360^{\circ}$ rotation of one of the ends of the string does not generate any phase. This is nothing but the spin-statistics theorem.

The emergence of Fermi statistics in the $\left|\Phi_{Z_{2}}\right\rangle$ state of a purely bosonic spin-1/2 model indicates that the state is a topologically ordered state. We also see that the $\left|\Phi_{Z_{2}}\right\rangle$ state has a bosonic quasiparticle |\rangle$_{\text {def }}+|\rangle_{\text {def, and a fermionic quasi- }}$ particle |\rangle$_{\text {def }}-|\rangle_{\text {def. }}$. The bound state of the above two particles is a boson (not a fermion) due to their mutual semion statistics. Such quasiparticle content agrees exactly with the $Z_{2}$ gauge theory which also has three types of nontrivial quasiparticles excitations, two bosons and one fermion. In fact, the low-energy effective theory of the topologically ordered state $\left|\Phi_{Z_{2}}\right\rangle$ is the $Z_{2}$ gauge theory, and we will call $\left|\Phi_{Z_{2}}\right\rangle$ a $Z_{2}$ topologically ordered state.

Next, let us consider the defects in the $\left|\Phi_{\text {Semi }}\right\rangle$ state. Now we show that

$$
|i\rangle_{\text {def }=}|i\rangle+|\dot{p}\rangle-|\dot{\beta}\rangle+\cdots
$$

and a similar expression for $\left|{ }^{9}\right\rangle$ def, due to a change of the local dancing rule for reconnecting the strings (see (6)). Using the string reconnection move in Figure 7 , we find that \langle\rangle$_{\text {def }}=-$

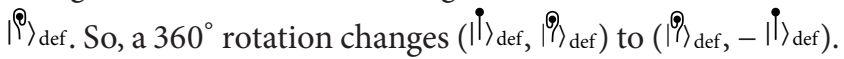
We find that |\rangle$_{\text {def }}+i|\rangle_{\text {def }}$ is the eigenstate of the $360^{\circ}$ rotation with eigenvalue $-i$, and $|i\rangle_{\text {def }}-i|\rangle_{\text {def }}$ is the other eigenstate of the $360^{\circ}$ rotation with eigenvalue $i$. So, the particle |\rangle$_{\text {def }}+i$ 9 $\rangle_{\text {def }}$ has a spin $-1 / 4$, and the particle |\rangle$_{\text {def }}-i|\rangle_{\text {def }}$ has a spin $1 / 4$. The spin-statistics theorem is still valid for $\left|\Phi_{\text {Semi }}\right\rangle_{\text {def }}$ state, as one can see from Figure 16. So, the particle $|i\rangle_{\text {def }}+i$ $|9\rangle_{\text {def }}$ and particle $|i\rangle_{\text {def }}-i\left|{ }^{9}\right\rangle_{\text {def }}$ have fractional statistics with statistical angles of semion: $\pm \pi / 2$. Thus, the $\left|\Phi_{\text {Semi }}\right\rangle$ state contains a nontrivial topological order. We will call such a topological order a double-semion topological order.

It is amazing to see that the long-range quantum entanglements in string liquid can give rise to fractional spin and fractional statistics, even from a purely bosonic model. Fractional 

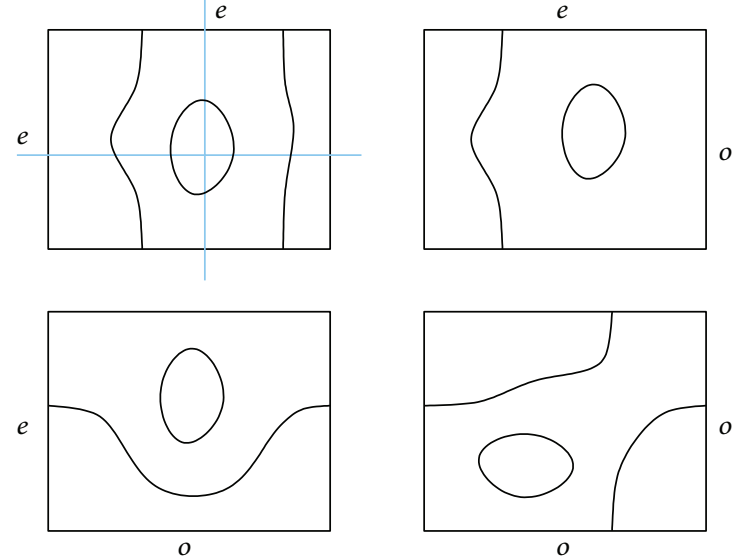

FIGURE 17: On a torus, the closed string configurations can be divided into four sectors, depending on even or odd number of strings crossing the $x$ - or $y$-axes.

spin and Fermi statistics are two of the most mysterious phenomena in nature. Now, we can understand them as merely a phenomenon of long-range quantum entanglements. They are no longer mysterious.

5.2.2. Topological Degeneracy. The $Z_{2}$ and the double-semion topological states (as well as many other topological states) have another important topological property: topological degeneracy $[6,7]$. Topological degeneracy is the ground-state degeneracy of a gapped many-body system that is robust against any local perturbations as long as the system size is large.

Topological degeneracy can be used as protected qubits which allow us to perform topological quantum computation [28]. It is believed that the appearance of the topological degeneracy implies the topological order (or long-range entanglements) in the ground state $[6,7]$. Many-body states with topological degeneracy are described by topological quantum field theory at low energies [8].

The simplest topological degeneracy appears when we put topologically ordered states on compact spaces with no boundary. We can use the global dancing pattern to understand the topological degeneracy. We know that the local dancing rules determine the global dancing pattern. On a sphere, the local dancing rules determine a unique global dancing pattern. So, the ground state is nondegenerate. However, on other compact spaces, there can be several global dancing patterns that all satisfy the local dancing rules. In this case, the ground state is degenerate.

For the $Z_{2}$ topological state on torus, the local dancing rule relates the amplitudes of the string configurations that differ by a string reconnection operation in Figure 7 . On a torus, the closed string configurations can be divided into four sectors (see Figure 17), depending on even or odd number of strings crossing the $x$ - or $y$-axes. The string reconnection move only connect the string configurations among each sector. So, the superposition of the string configurations in each sector represents a different global dancing pattern and a different degenerate ground state. Therefore, the local

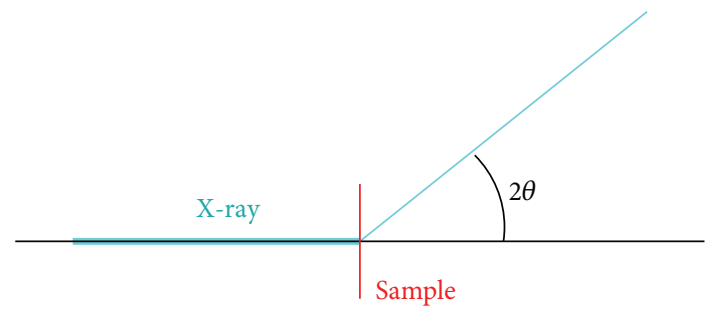

(a)

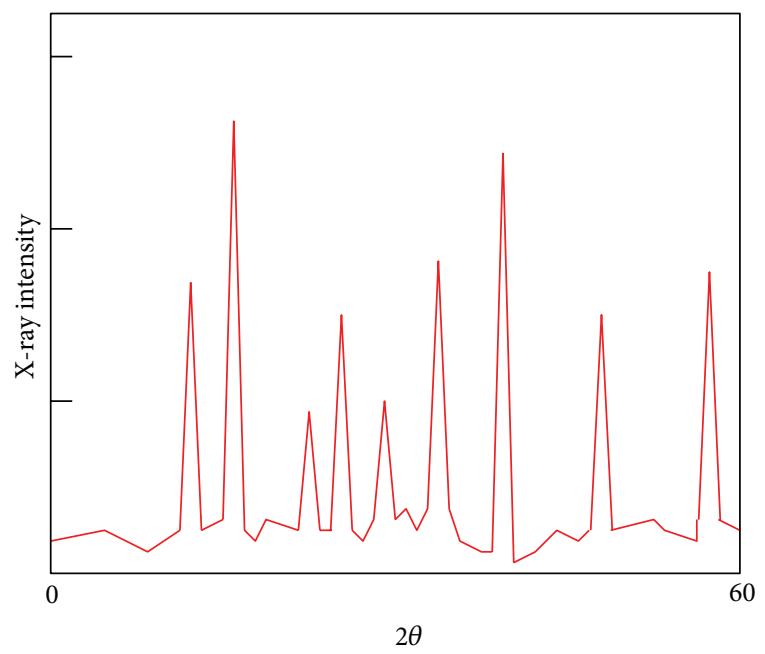

(b)

Figure 18: A X-ray diffraction pattern defines/probes the crystal order.

dancing rule for the $Z_{2}$ topological order gives rise to fourfold degenerate ground state on torus [23].

Similarly, the double-semion topological order also gives rise to fourfold degenerate ground state on torus.

\section{A Macroscopic Definition and the Characterization of Topological Order}

So far in this paper, we discussed topological order using an intuitive dancing picture. Then, we discussed a few simple examples. In the rest of this paper, we will give a more rigorous description and a systematic understanding of topological order and its essence [6,7]. Historically, the more rigorous description of topological order was obtained before the intuitive dancing picture and the simple examples of topological order discussed in the previous part of the paper.

First, we would like to give a physical definition of topological order (at least in $2+1$ dimensions). Here, we like to point out that to define a physical concept is to design experiments or numerical calculations that allow us to probe and characterize the concept. For example, the concept of superfluid order is defined by zero viscosity and the quantization of vorticity, and the concept of crystal order is defined by X-ray diffraction experiment (see Figure 18).

The experiments that we use to define/characterize superfluid order and crystal order are linear responses. Linear responses are easily accessible in experiments and 
TABLE 1: Symmetry-breaking orders can be probed/defined through linear responses. But topological order cannot be probed/defined through linear responses. We need topological probes to define topological orders.

\begin{tabular}{lc}
\hline Order & Experiment \\
\hline Crystal order & X-ray diffraction \\
Ferromagnetic order & Magnetization \\
Antiferromagnetic order & Neutron scattering \\
Superuid order & Zero viscosity and vorticity quantization \\
Topological order & Topological degeneracy \\
(Global dancing pattern) & non-Abelian geometric phase \\
\hline
\end{tabular}
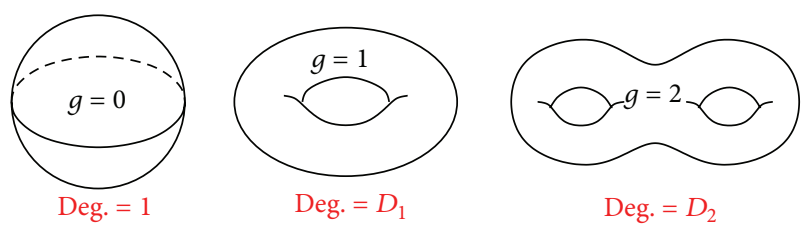

FIgURE 19: The topological ground state degeneracies of topologically ordered states depend on the topology of the space, such as the genus $g$ of two-dimensional closed surfaces.

the symmetry-breaking order that they define are easy to understand (see Table 1). However, topological order is such a new and elusive order that it cannot be probed/defined by any linear responses. To probe/define topological order we need to use very unusual "topological" probes. In 1990, we conjectured that topological order can be completely defined/ characterized by using only two topological properties (at least in $2+1$ dimensions) [7]:

(1) topological ground-state degeneracies on closed spaces of various topologies (see Figure 19) [6],

(2) non-Abelian geometric phases [71] of those degenerate ground states from deforming the spaces (see Figure 20) $[7,10]$.

It was through such topological probes that we introduce the concept of topological order. Just like zero viscosity and the quantization of vorticity define the concept of superfluid order, the topological degeneracy and the non-Abelian geometric phases of the degenerate ground states define the concept of topological order.

6.1. What Is "Topological Ground-State Degeneracy"? Topological ground state degeneracy, or simply, topological degeneracy is a phenomenon of quantum many-body systems, that the ground state of a gapped many-body system become degenerate in the large system size limit, and that such a degeneracy cannot be lifted by any local perturbations as long as the system size is large $[6,9,59,72]$. The topological degeneracy for a given system is usually different for different topologies of space [73]. For example, for the $Z_{2}$ topologically ordered state in two dimensions [5], the topological degeneracy is $D_{g}=4^{g}$ on genus $g$ Riemann surface (see Figure 19).

People usually attribute the ground-state degeneracy to symmetry. But topological degeneracy, being robust against

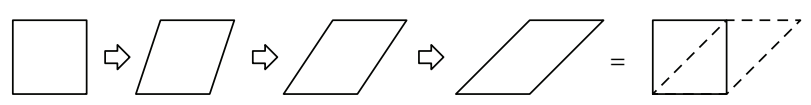

(a)

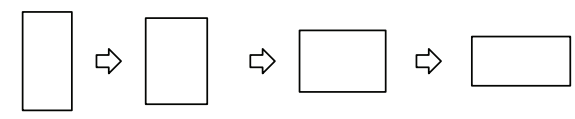

(b)

Figure 20: (a) The shear deformation of a torus generates a (projective) non-Abelian geometric phase $T$, which is a generator of a projective representation modular transformation. The last sheardeformed torus is the same as the original torus after a coordinate transformation: $x \rightarrow x+y, y \rightarrow y$. (b) The squeezing deformation of a torus generates a (projective) non-Abelian geometric phase $S$, which is the other generator of a projective representation modular transformation. The last squeeze-deformed torus is the same as the original torus after a coordinate transformation: $x \rightarrow y, y \rightarrow-x$.

any local perturbations, is not due to symmetry. So, the very existence of topological degeneracy is a surprising and amazing phenomenon. Such an amazing phenomenon defines the notion of topological order. As a comparison, we know that the existence of zero viscosity is also an amazing phenomenon, and such an amazing phenomenon defines the notion of superfluid order. So, topological degeneracy, playing the role of zero viscosity in superfluid order, implies the existence of a new kind of quantum phase-topologically ordered phases.

6.2. What Is "Non-Abelian Geometric Phase of Topologically Degenerate States"? However, the ground-state degeneracy is not enough to completely characterize/define topological order. Two different topological orders may have exactly the same topological degeneracy on space of any topology. We would like to find, as many as possible, quantum numbers associated with the degenerate ground states, so that by measuring these quantum numbers, we can completely characterize/define topological order. The non-Abelian geometric phases of topologically degenerate states are such quantum numbers $[7,10]$.

The non-Abelian geometric phase is a unitary matrix $U$ that can be calculated from an one parameter family of gapped Hamiltonians $H_{g}, g \in[0,1]$, provided that $H_{0}=H_{1}$ [71]. $U$ is a one by one matrix if there is only one ground state below the gap. $U$ is $n$ dimensional if the ground-state degeneracy is $n$ for all $g \in[0,1]$.

To use non-Abelian geometric phases to characterize/define topological order, let us put the many-body state on a torus $[7,10,74,75]$ and perform a "shear" deformation of the torus to obtain a one parameter family of gapped Hamiltonians that form a loop (i.e., $H_{0}=H_{1}$ ) (see Figure 20(a)). The non-Abelian geometric phase obtained this way is denoted as T. Similarly, a "squeezing" deformation of the torus gives rise to another non-Abelian geometric phase $S$. Both $S$ and $T$ are $D_{1}$ dimensional unitary matrices, where $D_{1}$ is the topological degeneracy on torus. For different deformation paths that realize the loops in Figure 20, $S$ and $T$ may be different. However, because the ground-state degeneracy is 
robust, the difference is only in the total phase factors. Since the two deformations in Figure 20 generate the modular transformations, thus $S$ and $T$ generate a projective representation of the modular transformations. It was conjectured that $S$ and $T$ (or the projective representation of the modular transformations) provide a complete characterization and definition of topological orders in $2+1$ dimensions $[7,10]$.

6.3. The Essence of Topological Orders. Yang once asked; the microscopic theory of fermionic superfluid and superconductor, BCS theory, captures the essence of the superfluid and superconductor, but what is this essence? This question led him to develop the theory of off-diagonal long-range order, [76] which reveals the essence of superfluid and superconductor. In fact, long-range order is the essence of any symmetry-breaking order.

Similarly, we may ask; Laughlin's theory for FQH effect captures the essence of the FQH effect, but what is this essence? Our answer is that the topological order defined by the topological ground-state degeneracy and the non-Abelian geometric phases of those degenerate ground states is the essence of FQH effect.

One may disagree with the above statement by pointing out that the essence of $\mathrm{FQH}$ effect should be the quantized Hall conductance. However, such an opinion is not quite correct, since even after we break the particle number conservation (which breaks the quantized Hall conductance), an FQH state is still a nontrivial state with a quantized thermal Hall conductance [77]. The nontrivialness of FQH state does not rely on any symmetry (except the conservation of energy). In fact, the topological degeneracy and the nonAbelian geometric phases discussed above are the essence of $\mathrm{FQH}$ states which can be defined even without any symmetry. They provide a characterization and definition of topological order that does not rely on any symmetry. We would like to point out that the topological entanglement entropy is another way to characterize the topological order without any symmetry $[78,79]$.

\section{The Microscopic Description of Topological Order}

After the experimental discovery of superconducting order via zero resistance and Meissner effect [80, 81], it took 40 years to obtain the microscopic understanding of superconducting order through the condensation of fermion pairs [82]. However, we are luckier for topological orders. After the theoretical discovery of topological order via the topological degeneracy and the non-Abelian geometric phases of the degenerate ground states [7], it took only 20 years to obtain the microscopic understanding of topological order; topological order is due to long-range entanglements, and topological order is simply a pattern of long-range entanglements [83]. In this section, we will explain such a microscopic understanding.

7.1. Local Unitary Transformations. The long-range entanglements are defined through local unitary (LU) transformations. LU transformation is an important concept which is

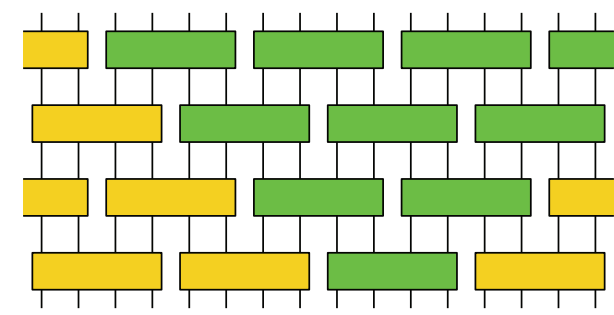

(a)

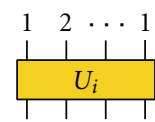

(b)
FIGURE 21: (a) A graphic representation of a quantum circuit, which is formed by (b) unitary operations on blocks of finite size $l$. The green shading represents a causal structure.

directly related to the definition of quantum phases [83]. In this section, we will give a short review of LU transformation [29, 83-85].

Let us first introduce local unitary evolution. An LU evolution is defined as the following unitary operator that acts on the degrees of freedom in a quantum system:

$$
\mathscr{T}\left[e^{-i \int_{0}^{1} d g \widetilde{H}(g)}\right],
$$

where $\mathscr{T}$ is the path-ordering operator and $\widetilde{H}(g)=\sum_{i} O_{i}(g)$ is a sum of local Hermitian operators. Two gapped quantum states belong to the same phase if and only if they are related by an LU evolution $[59,83,86]$.

The LU evolution is closely related to quantum circuits with finite depth. To define quantum circuits, let us introduce piecewise local unitary operators. A piecewise local unitary operator has a form

$$
U_{\mathrm{pwl}}=\prod_{i} U^{i}
$$

where $\left\{U^{i}\right\}$ is a set of unitary operators that act on nonoverlapping regions. The size of each region is less than some finite number $l$. The unitary operator $U_{\mathrm{pwl}}$ defined in this way is called a piecewise local unitary operator with range $l$. A quantum circuit with depth $M$ is given by the product of $M$ piecewise local unitary operators(see Figure 21):

$$
U_{\mathrm{circ}}^{M}=U_{\mathrm{pwl}}^{(1)} U_{\mathrm{pwl}}^{(2)} \cdots U_{\mathrm{pwl}}^{(M)} .
$$

We will call $U_{\text {circ }}^{M}$ an LU transformation. In quantum information theory, it is known that finite time unitary evolution with local Hamiltonian (LU evolution defined above) can be simulated with constant depth quantum circuit (i.e., an LU transformation) and vice versa:

$$
\mathscr{T}\left[e^{-i \int_{0}^{1} d g \widetilde{H}(g)}\right]=U_{\text {circ }}^{M} .
$$

So, two gapped quantum states belong to the same phase if and only if they are related by an LU transformation.

7.2. Topological Orders and Long-Range Entanglements. The notion of LU transformations leads to the following more general and more systematic picture of phases and phase 
transitions (see Figure 22) [83]. For gapped quantum systems without any symmetry, their quantum phases can be divided into two classes: short-range entangled (SRE) states and longrange entangled (LRE) states.

SRE states are states that can be transformed into direct product states via LU transformations. All SRE states can be transformed into each other via LU transformations. So, all SRE states belong to the same phase (see Figure 22).

LRE states are states that cannot be transformed into direct product states via LU transformations. It turns out that many LRE states also cannot be transformed into each other. The LRE states that are not connected via LU transformations belong to different classes and represent different quantum phases. Those different quantum phases are nothing but the topologically ordered phases. So, topological order is a pattern of long-range entanglements.

Such understanding of topological order in terms of longrange entanglements leads to a systematic description of boundary-gapped (BG) topological orders in $2+1$ dimensions $[29,83,87,88]$, in terms of spherical fusion category [70]. (Here, a BG topological order is a long-range entangled phase which can have a gapped edge or gapped entanglement spectrum [89].)

In $(2+1) D$, BG topological orders can be viewed as string-net liquids, where the global dancing patterns (i.e., topological orders or patterns of long-range entanglements) can be determined by local dancing rules that are similar to (5) and (6). For those more general BG topological orders, the strings in the string-net liquid may have several types labeled by $i, j, \ldots=0,1, \ldots, N$, and they may join to form a stringnet. The local dancing rules relate the amplitudes of string-net configurations that only differ by small local transformations. To write down a set of local rules, one first chooses a real tensor $d_{i}$ and a complex tensor $F_{k l n}^{i j m}$ where the indices $i, j$, $k, l, m$, and $n$ run over the different string types $0,1, \ldots, N$. The local dancing rules are then given by

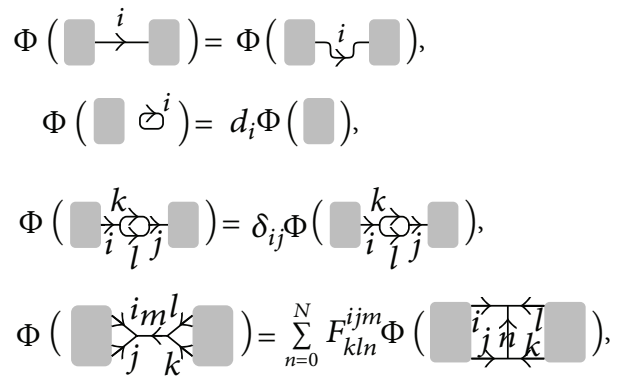

where the shaded areas represent other parts of string-nets that are not changed. Here, the type- 0 string is interpreted as the no-string state. We would like to mention that we have drawn the first local rule somewhat schematically. The more precise statement of this rule is that any two string-net configurations that can be continuously deformed into each other have the same amplitude. In other words, the stringnet wave function $\Phi$ only depends on the topologies of the graphs; it only depends on how the strings are connected (see Figure 12).

By applying the local rules in (13) multiple times, one can compute the amplitude of any string-net configuration in

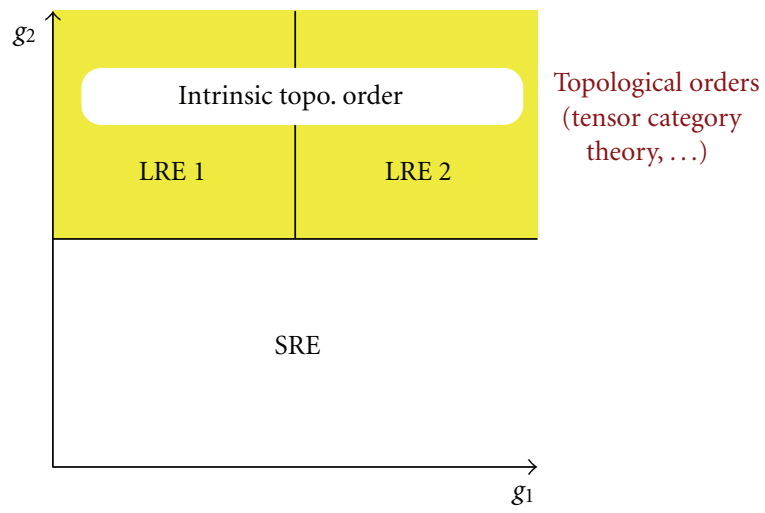

FIGURE 22: The possible gapped phases for a class of Hamiltonians $H\left(g_{1}, g_{2}\right)$ without any symmetry restriction. Each phase is labeled by its entanglement properties. SRE stands for short-range entanglement and LRE for long-range entanglement which correspond to topologically ordered phases.

terms of the amplitude of the no-string configuration. Thus, (13) determines the string-net wave function $\Phi$.

However, an arbitrary choice of $\left(d_{i}, F_{l m n}^{i j k}\right)$ does not lead to a well-defined $\Phi$. This is because two string-net configurations may be related by more than one sequence of local rules. We need to choose the $\left(d_{i}, F_{l m n}^{i j k}\right)$ carefully so that different sequences of local rules produce the same results. That is, we need to choose $\left(d_{i}, F_{l m n}^{i j k}\right)$, so that the rules are self-consistent. Finding these special tensors is the subject of tensor category theory $[90,91]$. It has been shown that only those that satisfy [29]

$$
\begin{gathered}
F_{j^{*} i^{*} 0}^{i j k}=\frac{v_{k}}{v_{i} v_{j}} \delta_{i j k}, \\
F_{k l n}^{i j m}=F_{j i n}^{l k m^{*}}=F_{l k n^{*}}^{j i m}=F_{k^{*} n l}^{i m j} \frac{v_{m} v_{n}}{v_{j} v_{l}} \\
\sum_{n=0}^{N} F_{k p n}^{m l q} F_{m n s}^{j i p} F_{l k r}^{j s n}=F_{q k r}^{j i p} F_{m l s}^{r i q}
\end{gathered}
$$

will result in self-consistent rules and a well-defined stringnet wave function $\Phi$. Such a wave function describes a stringnet condensed state. Here, we have introduced some new notation; $v_{i}$ is defined by $v_{i}=v_{i^{*}}=\sqrt{d_{i}}$ while $\delta_{i j k}$ is given by

$$
\delta_{i j k}= \begin{cases}1, & \text { if } i, j, k \text { strings can join, } \\ 0, & \text { otherwise }\end{cases}
$$

The solutions $\left(d_{i}, F_{l m n}^{i j k}\right)$ give us a quantitative description of topological orders (or pattern of long-range entanglements), in terms of local dancing rules. From the data $\left(d_{i}, F_{l m n}^{i j k}\right)$, we can compute the topological properties of the corresponding topological phases, such as ground-state degeneracy and quasiparticle statistics $[29,69,83,87,91,92]$. The above approach can also be used to systematically describe BG topological orders in $3+1$ dimensions $[29,36,93]$. 
We know that group theory is the mathematical foundation of symmetry-breaking theory of phases and phase transitions. The above systematic description of $(2+1) D$ BG topological order strongly suggests that tensor category theory is the mathematical foundation of topological order and long-range entanglements. Because of symmetry, group theory become very important in physics. Because of quantum entanglements, tensor category theory will become very important in physics.

\section{Where to Find Long-Range Entangled Quantum Matter?}

In this paper, we described the world of quantum phases. We pointed out that there are symmetry-breaking quantum phases and there are topologically ordered quantum phases. The topologically ordered quantum phases are a totally new kind of phases which cannot be understood using the conventional concepts (such as symmetry breaking, longrange order, and order parameter) and conventional mathematical framework (such as group theory and GinzburgLandau theory). The main goal of this paper is to introduce new concepts and pictures to describe the new topologically ordered quantum phases.

In particular, we described how to use global dancing pattern to gain an intuitive picture of topological order (which is a pattern of long-range entanglements). We further point out that we can use local dancing rules to quantitatively describe the global dancing pattern (or topological order). Such an approach leads to a systematic description of BG topological order in terms of string-net (or spherical fusion category theory) $[29,83,87,88]$ and systematic description of $2 \mathrm{D}$ chiral topological order in terms of pattern of zeros [94102] (which is a generalization of "CDW" description of FQH states [103-110]).

The local-dancing-rule approach also leads to concrete and explicit Hamiltonians that allow us to realize each stringnet state and each FQH state described by pattern of zeros. However, those Hamiltonians usually contain three-body or more complicated interactions and are hard to realize in real materials. So, here we would like to ask; can topological order be realized by some simple Hamiltonians and real materials?

Of cause, nontrivial topological orders-FQH statescan be realized by $2 \mathrm{D}$ electron gas under very strong magnetic fields and very low temperatures $[11,12]$. Recently, it was proposed that FQH states might appear even at room temperatures with no magnetic field in flat-band materials with spin-orbital coupling and spin polarization [111-115]. Finding such materials and realizing FQH states at high temperatures will be an amazing discovery. Using flat-band materials, we may even realize non-Abelian fractional quantum Hall states $[41,42,116,117]$ at high temperatures.

Apart from the FQH effects, nontrivial topological order may also appear in quantum spin systems. In fact, the concept of topological order was first introduced [6] to describe a chiral spin liquid $[4,5]$, which breaks time reversal and parity symmetry. Soon after, time reversal and parity symmetric topological order was proposed in 1991 [23, 25-27], which had spin-charge separation and emergent fermions. The new topological spin liquid is called $Z_{2}$ spin liquid or $Z_{2}$ topological order, since the low-energy effective theory is a $Z_{2}$ gauge theory. In 1997, an exactly soluble model [28] (that breaks the spin-rotation symmetry) was obtained that realizes the $Z_{2}$ topological order. Since then, the $Z_{2}$ topological order has become widely accepted.

More recently, extensive new numerical calculations indicated that the Heisenberg model on Kagome lattice [118-122]

$$
H=\sum_{\text {n.n. }} J \mathbf{S}_{i} \cdot \mathbf{S}_{j}
$$

and the $J_{1}-J_{2}$ model on square lattice [123-125]

$$
H=\sum_{\text {n.n. }} J_{1} \mathbf{S}_{i} \cdot \mathbf{S}_{j}+\sum_{\text {n.n.n. }} J_{2} \mathbf{S}_{i} \cdot \mathbf{S}_{j}, \quad \frac{J_{2}}{J_{1}} \sim 0.5
$$

may have gapped spin liquid ground states, and such spin liquids are very likely to be $Z_{2}$ spin liquids. However, with spin rotation, time reversal, and lattice symmetry, there are many $Z_{2}$ spin liquids [126-129]. It is not clear which $Z_{2}$ spin liquids are realized by the Heisenberg model on Kagome lattice and the $J_{1}-J_{2}$ model on square lattice.

The Heisenberg model on Kagome lattice can be realized in Herbertsmithite $\mathrm{ZnCu}_{3}(\mathrm{OH})_{6} \mathrm{Cl}_{2}[130,131]$. Although $J$ is as large as $150 \mathrm{~K}$, no spin ordering and other finite temperature phase transitions are found down to $50 \mathrm{mK}$. So, Herbertsmithite may realize a 2D spin-liquid state. However, experimentally, it is not clear if the spin liquid is a gapped spin liquid or a gapless spin liquid. Theoretically, both a gapped $Z_{2}$ spin liquid $[121,122,129,132]$ and a gapless $U(1)$ spin liquid [133-135] are proposed for the Heisenberg model on Kagome lattice. The theoretical study suggests that the spin-liquid state in Herbertsmithite may have some very interesting characteristic properties. A magnetic field in $z$ direction may induce a spin order in $x y$-plane [136], and an electron (or hole) doping may induce a charge $4 e$ topological superconductor [137].

To summarize, topological order and long-range entanglements give rise to new states of quantum matter. Topological order has many new emergent phenomena, such as emergent gauge theory, fractional charge, fractional statistics, non-Abelian statistics, and perfect conducting boundary. In particular, if we can realize a quantum liquid of oriented strings in certain materials, it will allow us to make artificial elementary particles (such as artificial photons and artificial electrons). So, we can actually create an artificial vacuum, and an artificial world for that matter, by making an oriented string-net liquid. This would be a fun experiment to do!

\section{A New Chapter in Physics}

Our world is rich and complex. When we discover the inner working of our world and try to describe it, we often find that we need to invent new mathematical language to describe our understanding and insight. For example, when Newton discovered his law of mechanics, the proper mathematical language was not invented yet. Newton (and Leibniz) had to develop calculus in order to formulate the law of mechanics. 
For a long time, we tried to use the theory of mechanics and calculus to understand everything in our world.

As another example, when Einstein discovered the general equivalence principle to describe gravity, he needed a mathematical language to describe his theory. In this case, the needed mathematics, Riemannian geometry, had been developed, which leaded to the theory of general relativity. Following the idea of general relativity, we developed the gauge theory. Both general relativity and gauge theory can be described by the mathematics of fiber bundles. Those advances led to a beautiful geometric understanding of our world based on quantum field theory, and we tried to understand everything in our world in terms of quantum field theory.

Now, I feel that we are at another turning point. In a study of quantum matter, we find that long-range entanglements can give rise to many new quantum phases. So long-range entanglements are natural phenomena that can happen in our world. They greatly expand our understanding of possible quantum phases and bring the research of quantum matter to a whole new level. To gain a systematic understanding of new quantum phases and long-range entanglements, we like to know what mathematical language should we use to describe long-range entanglements? The answer is not totally clear. But early studies suggest that tensor category and group cohomology should be a part of the mathematical framework that describes long-range entanglements. The further progresses in this direction will lead to a comprehensive understanding of long-range entanglements and topological quantum matter.

However, what is really exciting in the study of quantum matter is that it might lead to a whole new point of view of our world. This is because long-range entanglements can give rise to both gauge interactions and Fermi statistics. In contrast, the geometric point of view can only lead to gauge interactions. So, maybe we should not use geometric pictures, based on fields and fiber bundles, to understand our world. Maybe we should use entanglement pictures to understand our world. This way, we can get both gauge interactions and fermions from a single origin-qubits. We may live in a truly quantum world. So, quantum entanglements represent a new chapter in physics.

\section{References}

[1] L. D. Landau, "Theory of phase transformations," Physikalische Zeitschrift der Sowjetunion, vol. 11, p. 26, 1937.

[2] V. L. Ginzburg and L. D. Landau, "On the theory of superconductivity," Zhurnal EksperImental'NoI I TeoretIcheskoI FIzIkI, vol. 20, pp. 1064-1082, 1950.

[3] L. D. Landau and E. M. Lifschitz, Statistical Physics-Course of Theoretical Physics, vol. 5, Pergamon, London, UK, 1958.

[4] V. Kalmeyer and R. B. Laughlin, "Equivalence of the resonatingvalence-bond and fractional quantum Hall states," Physical Review Letters, vol. 59, pp. 2095-2098, 1987.

[5] X. G. Wen, F. Wilczek, and A. Zee, "Chiral spin states and superconductivity," Physical Review B, vol. 39, no. 16, pp. 1141311423, 1989.

[6] X. G. Wen, "Vacuum degeneracy of chiral spin states in compactified space," Physical Review B, vol. 40, pp. 7387-7390, 1989.
[7] X. G. Wen, “Topological orders in rigid states," International Journal of Modern Physics B, vol. 4, p. 239, 1990.

[8] E. Witten, "Quantum field theory and the Jones polynomial," Communications in Mathematical Physics, vol. 121, no. 3, pp. 351399, 1989.

[9] X. G. Wen and Q. Niu, "Ground-state degeneracy of the fractional quantum Hall states in the presence of a random potential and on high-genus Riemann surfaces," Physical Review B, vol. 41, pp. 9377-9396, 1990.

[10] E. Keski-Vakkuri and X. G. Wen, "the ground state structure and modular transformations of fractional quantum hall states on a torus," International Journal of Modern Physics B, vol. 7, p. 4227, 1993.

[11] D. C. Tsui, H. L. Stormer, and A. C. Gossard, "Two-dimensional magnetotransport in the extreme quantum limit," Physical Review Letters, vol. 48, no. 22, pp. 1559-1562, 1982.

[12] R. B. Laughlin, "Anomalous quantum hall effect: an incompressible quantum fluid with fractionally charged excitations," Physical Review Letters, vol. 50, pp. 1395-1398, 1983.

[13] S. M. Girvin and A. H. MacDonald, "Off-diagonal long-range order, oblique confinement, and the fractional quantum Hall effect," Physical Review Letters, vol. 58, pp. 1252-1255, 1987.

[14] N. Read, "Order parameter and Ginzburg-Landau theory for the fractional quantum Hall effect," Physical Review Letters, vol. 62, pp. 86-89, 1989.

[15] S. C. Zhang, T. H. Hansson, and S. Kivelson, "Effective-fieldtheory model for the fractional quantum Hall effect," Physical Review Letters, vol. 62, pp. 82-85, 1989.

[16] Z. F. Ezawa and A. Iwazaki, "Chern-Simons gauge theories for the fractional-quantum-Hall-effect hierarchy and anyon superconductivity," Physical Review B, vol. 43, no. 4, pp. 2637-2641, 1991.

[17] B. Blok and X. G. Wen, "Effective theories of the fractional quantum Hall effect: Hierarchy construction," Physical Review $B$, vol. 42, pp. 8145-8156, 1990.

[18] J. Frohlich and A. Zee, "Large scale physics of the quantum hall fluid," Nuclear Physics B, vol. 364, pp. 517-540, 1991.

[19] J. Frohlich and T. Kerler, "Universality in quantum Hall systems," Nuclear Physics B, vol. 354, pp. 369-417, 1991.

[20] X. G. Wen and A. Zee, "Classification of Abelian quantum Hall states and matrix formulation of topological fluids," Physical Review B, vol. 46, pp. 2290-2301, 1992.

[21] J. Frohlich and U. M. Studer, "Gauge invariance and current algebra in nonrelativistic many-body theory," Reviews of Modern Physics, vol. 65, pp. 733-802, 1993.

[22] X. G. Wen, “Topological orders and Chern-Simons theory in strongly correlated quantum liquid," International Journal of Modern Physics B, vol. 5, no. 10, p. 1641, 1991.

[23] X. G. Wen, "Mean-field theory of spin-liquid states with finite energy gap and topological orders," Physical Review B, vol. 44, pp. 2664-2672, 1991.

[24] T. H. Hansson, V. Oganesyan, and S. L. Sondhi, "Superconductors are topologically ordered," Annals of Physics, vol. 313, no. 2, pp. 497-538, 2004.

[25] N. Read and S. Sachdev, "Large- $N$ expansion for frustrated quantum antiferromagnets," Physical Review Letters, vol. 66, pp. 1773-1776, 1991.

[26] G. Misguich, C. Lhuillier, B. Bernu, and C. Waldtmann, "Spinliquid phase of the multiple-spin exchange Hamiltonian on the triangular lattice," Physical Review B, vol. 60, no. 2, pp. 10641074, 1999. 
[27] R. Moessner and S. L. Sondhi, "Resonating valence bond phase in the triangular lattice quantum Dimer model," Physical Review Letters, vol. 86, pp. 1881-1884, 2001.

[28] A. Y. Kitaev, "Fault-tolerant quantum computation by anyons," Annals of Physics, vol. 303, pp. 2-30, 2003.

[29] M. Levin and X. G. Wen, "String-net condensation: a physical mechanism for topological phases," Physical Review B, vol. 71, Article ID 045110, 21 pages, 2005.

[30] X. G. Wen, "Origin of gauge Bosons from strong quantum correlations," Physical Review Letters, vol. 88, Article ID 011602, 4 pages, 2002.

[31] T. Senthil and O. Motrunich, "Microscopic models for fractionalized phases in strongly correlated systems," Physical Review B, vol. 66, Article ID 205104, 9 pages, 2002.

[32] X. G. Wen, "Artificial light and quantum order in systems of screened dipoles," Physical Review B, vol. 68, Article ID 115413, 12 pages, 2003.

[33] X. G. Wen, "Quantum order from string-net condensations and the origin of light and massless fermions," Physical Review D, vol. 68, Article ID 065003, 25 pages, 2003.

[34] R. Moessner and S. L. Sondhi, "Three-dimensional resonatingvalence-bond liquids and their excitations," Physical Review B, vol. 68, Article ID 184512, 8 pages, 2003.

[35] M. Hermele, M. P. A. Fisher, and L. Balents, "Pyrochlore photons: the $U(1)$ spin liquid in a $s=1 / 2$ three-dimensional frustrated magnet," Physical Review B, vol. 69, no. 6, Article ID 064404, 21 pages, 2004.

[36] M. A. Levin and X. G. Wen, "Colloquium: photons and electrons as emergent phenomena," Reviews of Modern Physics, vol. 77, pp. 871-879, 2005.

[37] M. Levin and X. G. Wen, "Quantum ether: photons and electrons from a rotor model," Physical Review B, vol. 73, Article ID 035122, 10 pages, 2006.

[38] C. Castelnovo, R. Moessner, and S. L. Sondhi, "Spin ice, fractionalization, and topological order," Annual Review of Condensed Matter Physics, vol. 3, pp. 35-55, 2012.

[39] B. I. Halperin, "Statistics of quasiparticles and the hierarchy of fractional quantized Hall states," Physical Review Letters, vol. 52, pp. 1583-1586, 1984.

[40] D. Arovas, J. R. Schrieffer, and F. Wilczek, "Fractional statistics and the quantum Hall effect," Physical Review Letters, vol. 53, pp. 722-723, 1984.

[41] X. G. Wen, "Non-Abelian statistics in the fractional quantum Hall states," Physical Review Letters, vol. 66, pp. 802-805, 1991.

[42] G. Moore and N. Read, "Nonabelions in the fractional quantum hall effect," Nuclear Physics B, vol. 360, no. 2-3, pp. 362-396, 1991.

[43] R. Jackiw and C. Rebbi, "Solitons with fermion number," Physical Review D, vol. 13, no. 12, pp. 3398-3409, 1976.

[44] E. Dennis, A. Kitaev, A. Landahl, and J. Preskill, “Topological quantum memory," Journal of Mathematical Physics, vol. 43, no. 9, pp. 4452-4505, 2002.

[45] B. I. Halperin, "Quantized Hall conductance, current-carrying edge states, and the existence of extended states in a twodimensional disordered potential," Physical Review B, vol. 25, pp. 2185-2190, 1982.

[46] X. G. Wen, "Gapless boundary excitations in the quantum Hall states and in the chiral spin states," Physical Review B, vol. 43, pp. 11025-11036, 1991.
[47] A. H. MacDonald, "Edge states in the fractional-quantum-Halleffect regime," Physical Review Letters, vol. 64, pp. 220-223, 1990.

[48] K. von Klitzing, G. Dorda, and M. Pepper, "New method for high-accuracy determination of the fine-structure constant based on quantized hall resistance," Physical Review Letters, vol. 45, pp. 494-497, 1980.

[49] M. Levin and X. G. Wen, "Fermions, strings, and gauge fields in lattice spin models," Physical Review B, vol. 67, Article ID 245316, 10 pages, 2003.

[50] A. D'Adda, P. D. Vecchia, and M. Luscher, "A $1 / n$ expandable series of non-linear $\sigma$ models with instantons," Nuclear Physics $B$, vol. 146, no. 1, pp. 63-76, 1978.

[51] E. Witten, "Instatons, the quark model, and the $1 / N$ expansion," Nuclear Physics B, vol. 149, no. 2, pp. 285-320, 1979.

[52] G. Baskaran and P. W. Anderson, "Gauge theory of hightemperature superconductors and strongly correlated Fermi systems," Physical Review B, vol. 37, pp. 580-583, 1988.

[53] I. Aeck and J. B. Marston, "Large- $n$ limit of the HeisenbergHubbard model: implications for high- $T_{c}$ superconductors," Physical Review B, vol. 37, pp. 3774-3777, 1988.

[54] K. G. Wilson, “Confinement of quarks," Physical Review D, vol. 10, pp. 2445-2459, 1974.

[55] J. Kogut and L. Susskind, "Hamiltonian formulation of Wilson's lattice gauge theories," Physical Review D, vol. 11, no. 2, pp. 395408, 1975.

[56] T. Banks, R. Myerson, and J. Kogut, "Phase transitions in Abelian lattice gauge theories," Nuclear Physics, Section B, vol. 129, no. 3, pp. 493-510, 1977.

[57] J. B. Kogut, "An introduction to lattice gauge theory and spin systems," Reviews of Modern Physics, vol. 51, pp. 659-713, 1979.

[58] R. Savit, "Duality in field theory and statistical systems," Reviews of Modern Physics, vol. 52, pp. 453-487, 1980.

[59] M. B. Hastings and X. G. Wen, "Quasiadiabatic continuation of quantum states: the stability of topological ground-state degeneracy and emergent gauge invariance," Physical Review B, vol. 72, Article ID 045141, 14 pages, 2005.

[60] D. Foerster, H. B. Nielsen, and M. Ninomiya, "Dynamical stability of local gauge symmetry creation of light from chaos," Physics Letters B, vol. 94, p. 135, 1980.

[61] O. I. Motrunich and T. Senthil, "Exotic order in simple models of Bosonic systems," Physical Review Letters, vol. 89, Article ID 277004, 4 pages, 2002.

[62] J. M. Leinaas and J. Myrheim, "On the theory of identical particles," Il Nuovo Cimento B Series 11, vol. 37, no. 1, pp. 1-23, 1977.

[63] F. Wilczek, "quantum mechanics of fractional-spin particles," Physical Review Letters, vol. 49, pp. 957-959, 1982.

[64] I. Tamm, "Die verallgemeinerten kugelfunktionen und die wellenfunktionen eines elektrons im felde eines magnetpoles," Zeitschrift für Physik, vol. 71, no. 3-4, pp. 141-150, 1931.

[65] R. Jackiw and C. Rebbi, "Spin from isospin in a gauge theory," Physical Review Letters, vol. 36, no. 19, pp. 1116-1119, 1976.

[66] F. Wilczek, "Remarks on Dyons," Physical Review Letters, vol. 48, pp. 1146-1149, 1982.

[67] A. S. Goldhaber, "Electromagnetism, spin, and statistics," Physical Review Letters, vol. 49, pp. 905-908, 1982.

[68] K. Lechner and P. A. Marchetti, "Spin-statistics transmutation in relativistic quantum field theories of dyons," Journal of High Energy Physics, vol. 2000, 2000. 
[69] K. Walker, Z. Wang, L. Fidkowski, M. Freedman, and C. Nayak, "From String Nets to Nonabelions," Communications in Mathematical Physics, vol. 287, no. 3, pp. 805-827, 2009.

[70] Z. Wang, Topological Quantum Computation, CBMS Regional Conference Series in Mathematics, American Mathematical Society, 2010.

[71] F. Wilczek and A. Zee, "appearance of gauge structure in simple dynamical systems," Physical Review Letters, vol. 52, pp. 21112114, 1984.

[72] X. G. Wen and A. Zee, "Topological degeneracy of quantum Hall fluids," Physical Review B, vol. 58, no. 23, pp. 15717-15728, 1998.

[73] F. D. M. Haldane and E. H. Rezayi, "Periodic Laughlin-Jastrow wave functions for the fractional quantized Hall effect," Physical Review B, vol. 31, no. 4, pp. 2529-2531, 1985.

[74] Y. Zhang, T. Grover, A. Turner, M. Oshikawa, and A. Vishwanath, "Quasiparticle statistics and braiding from groundstate entanglement," Physical Review B, vol. 85, Article ID 235151, 15 pages, 2012.

[75] Y. Zhang and A. Vishwanath, "Establishing non-Abelian topological order in Gutzwiller projected Chern insulators via Entanglement Entropy and Modular S-matrix," http://arxiv.org/ abs/1209.2424.

[76] C. N. Yang, "Concept of off-diagonal long-range order and the quantum phases of liquid he and of superconductors," Reviews of Modern Physics, vol. 34, pp. 694-704, 1962.

[77] C. L. Kane and M. P. A. Fisher, "Quantized thermal transport in the fractional quantum Hall effect," Physical Review B, vol. 55, pp. 15832-15837, 1997.

[78] A. Kitaev and J. Preskill, "Topological entanglement entropy," Physical Review Letters, vol. 96, Article ID 110404, 4 pages, 2006.

[79] M. Levin and X. G. Wen, "Detecting topological order in a ground state wave function," Physical Review Letters, vol. 96, Article ID 110405, 4 pages, 2006.

[80] H. K. Onnes, "Further experiments with liquid helium. C. On the change of electric resistance of pure metals at very low temperatures, etc. IV. The resistance of pure mercury at helium temperatures," Communications from the Physical Laboratory at the University of Leiden, no. 120b, 1911.

[81] H. K. Onnes, "Further experiments with liquid helium. D. On the change of electric resistance of pure metals at very low temperatures, etc. V. The disappearance of the resistance of mercury," Communications from the Physical Laboratory at the University of Leiden, no. 122b, 1911.

[82] J. Bardeen, L. N. Cooper, and J. R. Schrieer, "Theory of superconductivity," Physical Review, vol. 108, pp. 1175-1204, 1957.

[83] X. Chen, Z. C. Gu, and X. G. Wen, "Local unitary transformation, long-range quantum entanglement, wave function renormalization, and topological order," Physical Review B, vol. 82, Article ID 155138, 28 pages, 2010.

[84] F. Verstraete, J. I. Cirac, J. I. Latorre, E. Rico, and M. M. Wolf, "Renormalization-group transformations on quantum states," Physical Review Letters, vol. 94, no. 14, Article ID 140601, 4 pages, 2005.

[85] G. Vidal, "Entanglement renormalization," Physical Review Letters, vol. 99, no. 22, Article ID 220405, 4 pages, 2007.

[86] S. Bravyi, M. B. Hastings, and S. Michalakis, "Topological quantum order: stability under local perturbations," Journal of Mathematical Physics, vol. 51, no. 9, Article ID 093512, 33 pages, 2010.
[87] S. M. Hong, "On symmetrization of 6j-symbols and Levin-Wen Hamiltonian," http://arxiv.org/abs/0907.2204.

[88] Z.-C. Gu, Z. Wang, and X.-G. Wen, "A classification of 2D fermionic and bosonic topological orders," http://arxiv.org/abs/ 1010.1517.

[89] H. Li and F. D. M. Haldane, "entanglement spectrum as a generalization of entanglement entropy: identification of topological order in non-abelian fractional quantum hall effect states," Physical Review Letters, vol. 101, Article ID 010504, 4 pages.

[90] V. G. Turaev, Quantum Invariants of Knots and 3-Manifolds, De Gruyter, New York, NY, USA, 1994.

[91] M. Freedman, C. Nayak, K. Shtengel, K. Walker, and Z. Wang, "A class of $P, T$-invariant topological phases of interacting electrons," Annals of Physics, vol. 310, no. 2, pp. 428-492, 2004.

[92] Y. Hu, S. D. Stirling, and Y.-S. Wu, "Ground-state degeneracy in the Levin-Wen model for topological phases," Physical Review $B$, vol. 85, no. 7, Article ID 075107, 8 pages, 2012.

[93] K. Walker and Z. Wang, "(3+1)-TQFTs and topological insulators," Frontiers of Physics, vol. 7, no. 2, pp. 150-159, 2012.

[94] X. G. Wen and Z. Wang, "Classification of symmetric polynomials of infinite variables: construction of Abelian and nonAbelian quantum Hall states," Physical Review B, vol. 77, Article ID 235108, 21 pages, 2008.

[95] X. G. Wen and Z. Wang, "Topological properties of Abelian and non-Abelian quantum Hall states classified using patterns of zeros," Physical Review B, vol. 78, Article ID 155109, 27 pages, 2008.

[96] N. Read, "Wavefunctions and counting formulas for quasiholes of clustered quantum Hall states on a sphere," Physical Review B, vol. 73, no. 24, Article ID 245334, 8 pages, 2006.

[97] S. H. Simon, E. H. Rezayi, and N. R. Cooper, "Pseudopotentials for multiparticle interactions in the quantum Hall regime," Physical Review B, vol. 75, no. 19, Article ID 195306, 11 pages, 2007.

[98] B. A. Bernevig and F. D. M. Haldane, "Model fractional quantum Hall states and Jack polynomials," Physical Review Letters, vol. 100, no. 24, Article ID 246802, 4 pages, 2008.

[99] B. A. Bernevig and F. D. M. Haldane, "Generalized clustering conditions of Jack polynomials at negative Jack parameter $\alpha$," Physical Review B, vol. 77, Article ID 184502, 10 pages, 2008.

[100] M. Barkeshli and X. G. Wen, "Structure of quasiparticles and their fusion algebra in fractional quantum Hall states," Physical Review B, vol. 79, Article ID 195132, 16 pages, 2009.

[101] M. Barkeshli and X. G. Wen, "Classification of Abelian and nonAbelian multilayer fractional quantum Hall states through the pattern of zeros," Physical Review B, vol. 82, Article ID 245301, 19 pages, 2010.

[102] Y. M. Lu, X. G. Wen, Z. Wang, and Z. Wang, "Non-Abelian quantum Hall states and their quasiparticles: from the pattern of zeros to vertex algebra," Physical Review B, vol. 81, Article ID 115124, 42 pages, 2010.

[103] A. Seidel and D. H. Lee, "Abelian and non-Abelian Hall liquids and charge-density wave: quantum number fractionalization in one and two dimensions," Physical Review Letters, vol. 97, Article ID 056804, 4 pages, 2006.

[104] E. J. Bergholtz, J. Kailasvuori, E. Wikberg, T. H. Hansson, and A. Karlhede, "Pfaffian quantum Hall state made simple: multiple vacua and domain walls on a thin torus," Physical Review B, vol. 74, no. 8, Article ID 081308, 2006.

[105] A. Seidel and D. H. Lee, "Domain-wall-type defects as anyons in phase space," Physical Review B, vol. 76, Article ID 155101, 12 pages, 2007. 
[106] A. Seidel, "Pfaffian statistics through adiabatic transport in the 1D coherent state representation," Physical Review Letters, vol. 101, Article ID 196802, 4 pages, 2008.

[107] A. Seidel and K. Yang, "Halperin $\left(m, m^{\prime}, n\right)$ Bilayer quantum Hall States on thin cylinders," Physical Review Letters, no. 3, 4 pages, 2008.

[108] E. Ardonne, E. J. Bergholtz, J. Kailasvuori, and E. Wikberg, "Degeneracy of non-Abelian quantum Hall states on the torus: domain walls and conformal field theory," Journal of Statistical Mechanics: Theory and Experiment, vol. 2008, no. 4, Article ID P04016, 2008.

[109] A. Seidel, "S-Duality constraints on 1D patterns associated with fractional quantum Hall states," Physical Review Letters, vol. 105, Article ID 026802, 4 pages, 2010.

[110] J. Flavin and A. Seidel, "Abelian and non-Abelian statistics in the coherent state representation," Physical Review X, vol. 1, no. 2, Article ID 021015, 37 pages, 2011.

[111] E. Tang, J. W. Mei, and X. G. Wen, "High-temperature fractional quantum Hall states," Physical Review Letters, vol. 106, Article ID 236802, 4 pages, 2011.

[112] K. Sun, Z. C. Gu, H. Katsura, and S. D. Sarma, "Nearly flatbands with nontrivial topology," Physical Review Letters, vol. 106, Article ID 236803, 4 pages, 2011.

[113] T. Neupert, L. Santos, C. Chamon, and C. Mudry, "Fractional quantum Hall states at zero magnetic field," Physical Review Letters, vol. 106, Article ID 236804, 4 pages, 2011.

[114] D. N. Sheng, Z. C. Gu, K. Sun, and L. Sheng, "Fractional quantum Hall effect in the absence of Landau levels," Nature Communications, vol. 2, article 389, 2011.

[115] A. G. Grushin, T. Neupert, C. Chamon, and C. Mudry, "Enhancing the stability of a fractional Chern insulator against competing phases," Physical Review B, vol. 86, Article ID 205125, 10 pages, 2012.

[116] R. Willett, J. P. Eisenstein, H. L. Strormer, D. C. Tsui, A. C. Gossard, and J. H. English, "Observation of an evendenominator quantum number in the fractional quantum Hall effect Observation of an even-denominator quantum number in the fractional quantum Hall effect," Physical Review Letters, vol. 59, pp. 1776-1779, 1987.

[117] I. P. Radu, J. B. Miller, C. M. Marcus, M. A. Kastner, L. N. Pfeiffer, and K. W. West, "Quasi-particle properties from tunneling in the $v=5 / 2$ fractional quantum hall state," Science, vol. 320, no. 5878, pp. 899-902, 2008.

[118] P. Lecheminant, B. Bernu, C. Lhuillier, L. Pierre, and P. Sindzingre, "Order versus disorder in the quantum Heisenberg antiferromagnet on the kagomé lattice using exact spectra analysis," Physical Review B, vol. 56, no. 5, pp. 2521-2529, 1997.

[119] C. Waldtmann, H. U. Everts, B. Bernu et al., "First excitations of the spin $1 / 2$ Heisenberg antiferromagnet on the kagomé lattice," European Physical Journal B, vol. 2, no. 4, pp. 501-507, 1998.

[120] P. W. Leung and V. Elser, "Numerical studies of a 36-site kagome antiferromagnet," Physical Review B, vol. 47, no. 9, pp. 5459$5462,1993$.

[121] H. C. Jiang, Z. Y. Weng, and D. N. Sheng, "Density matrix renormalization group numerical study of the kagome antiferromagnet," Physical Review Letters, vol. 101, no. 11, Article ID 117203, 4 pages, 2008.

[122] S. Yan, D. A. Huse, and S. R. White, "Spin-liquid ground state of the $S=1 / 2$ Kagome Heisenberg antiferromagnet," Science, vol. 332, no. 6034, pp. 1173-1176, 2011.
[123] H. C. Jiang, H. Yao, and L. Balents, "Spin liquid ground state of the spin-1/2 square $J_{1}-J_{2}$ Heisenberg model," Physical Review $B$, vol. 86, Article ID 024424, 14 pages, 2012.

[124] L. Wang, Z. C. Gu, F. Verstraete, and X. G. Wen, "Spin-liquid phase in spin-1/2 square $J_{1}-J_{2}$ Heisenberg model: a tensor product state approach," http://arxiv.org/abs/1112.3331.

[125] H. C. Jiang, Z. Wang, and L. Balents, "Identifying topological order by entanglement entropy," Nature Physics, vol. 8, pp. 902905, 2012.

[126] X. G. Wen, "Quantum orders and symmetric spin liquids," Physical Review B, vol. 65, no. 16, Article ID 165113, 37 pages, 2002.

[127] S. P. Kou, M. Levin, and X. G. Wen, "Mutual Chern-Simons theory for $Z_{2}$ topological order," Physical Review B, vol. 78, Article ID 155134, 13 pages, 2008.

[128] S. P. Kou and X. G. Wen, "Translation-symmetry-protected topological orders in quantum spin systems," Physical Review $B$, vol. 80, Article ID 224406, 11 pages, 2009.

[129] Y. M. Lu and Y. Ran, " $Z_{2}$ spin liquid and chiral antiferromagnetic phase in the Hubbard model on a honeycomb lattice," Physical Review B, vol. 84, Article ID 024420, 15 pages, 2011.

[130] J. S. Helton, K. Matan, M. P. Shores et al., "Spin dynamics of the spin-1/2 Kagome lattice antiferromagnet $\mathrm{ZnCu}_{3}(\mathrm{OH})_{6} \mathrm{Cl}_{2}$," Physical Review Letters, vol. 98, no. 10, Article ID 107204, 4 pages, 2007.

[131] T. Imai, M. Fu, T. H. Han, and Y. S. Lee, "Local spin susceptibility of the $S=1 / 2$ kagome lattice in $\mathrm{ZnCu}_{3}(\mathrm{OH})_{6} \mathrm{Cl}_{2}$," Physical Review B, vol. 84, Article ID 020411, 4 pages, 2011.

[132] Y. M. Lu, Y. Ran, and P. A. Lee, " $Z_{2}$ spin liquids in the $S=1 / 2$ Heisenberg model on the kagome lattice: a projective symmetry-group study of Schwinger fermion mean-field states," Physical Review B, vol. 83, no. 22, Article ID 224413, 11 pages, 2011.

[133] M. B. Hastings, "Dirac structure, RVB, and Goldstone modes in the Kagomé antiferromagnet," Physical Review B, vol. 63, no. 1, Article ID 14413, 16 pages, 2000.

[134] Y. Ran, M. Hermele, P. A. Lee, and X. G. Wen, "Projected-wavefunction study of the spin-1/2 Heisenberg model on the Kagomé Lattice," Physical Review Letters, vol. 98, no. 11, Article ID 117205, 4 pages, 2007.

[135] M. Hermele, Y. Ran, P. A. Lee, and X. G. Wen, "Properties of an algebraic spin liquid on the kagome lattice," Physical Review B, vol. 77, no. 22, Article ID 224413, 23 pages, 2008.

[136] Y. Ran, W. H. Ko, P. A. Lee, and X. G. Wen, "Spontaneous spin ordering of a dirac spin liquid in a magnetic field," Physical Review Letters, vol. 102, no. 4, Article ID 047205, 4 pages, 2009.

[137] W. H. Ko, P. A. Lee, and X. G. Wen, "Doped kagome system as exotic superconductor," Physical Review B, vol. 79, Article ID 214502, 13 pages, 2009. 

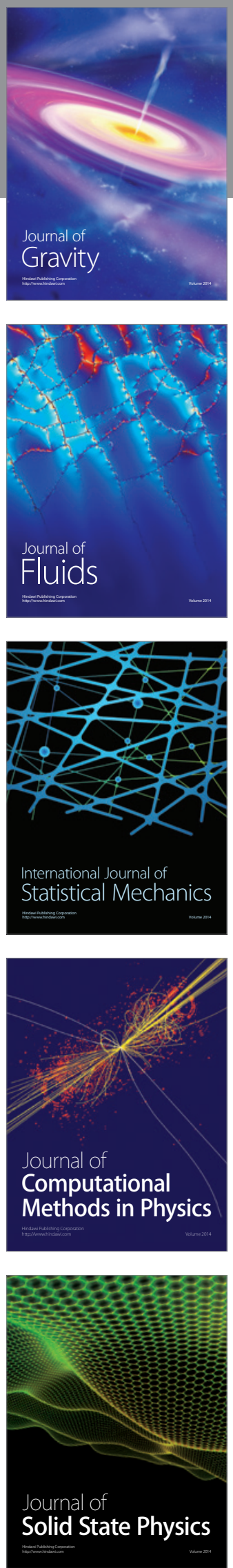

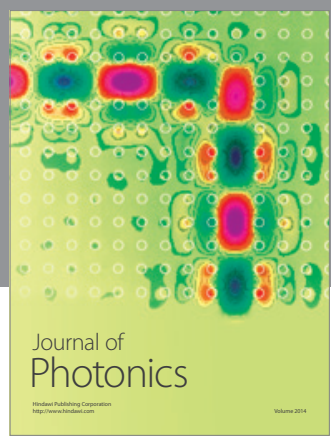

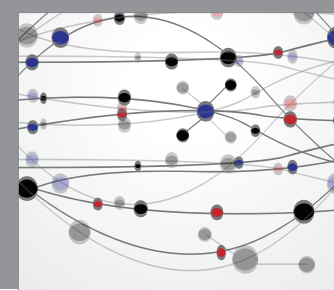

The Scientific World Journal

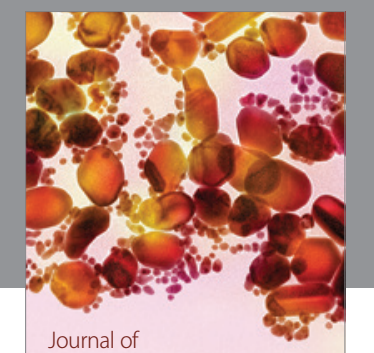

Soft Matter
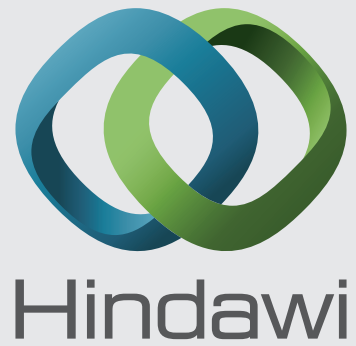

Submit your manuscripts at

http://www.hindawi.com
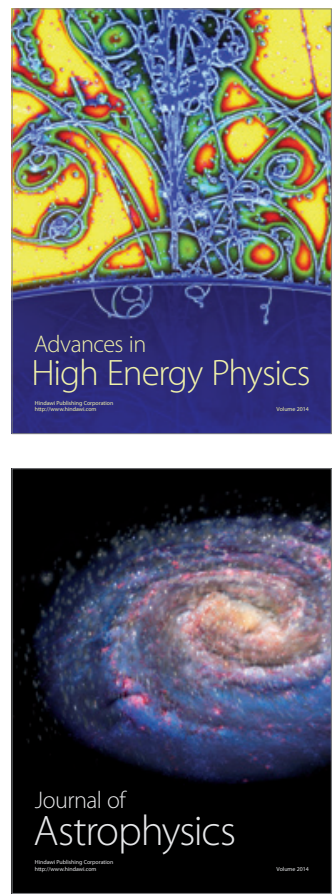
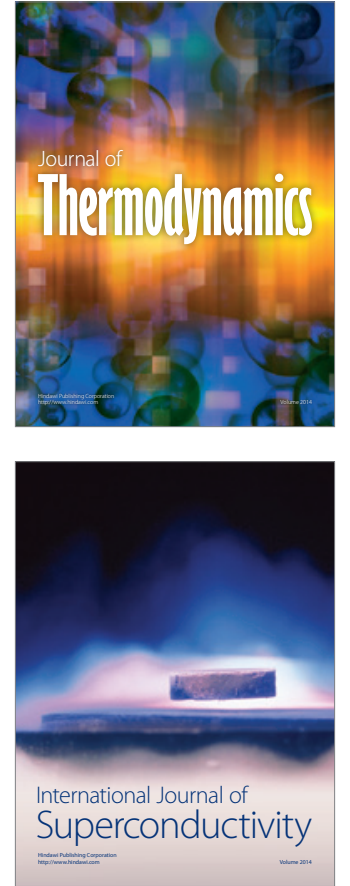
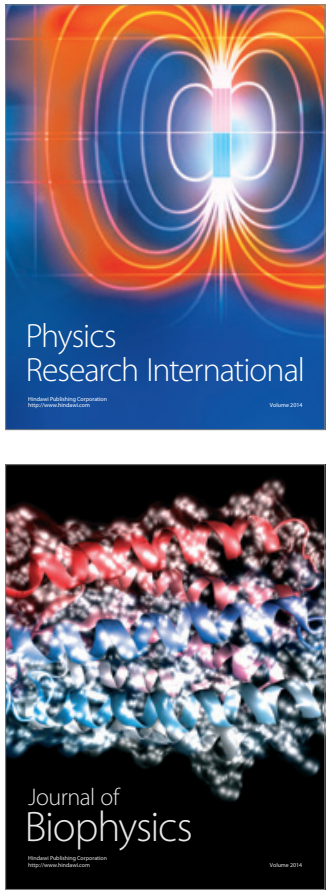
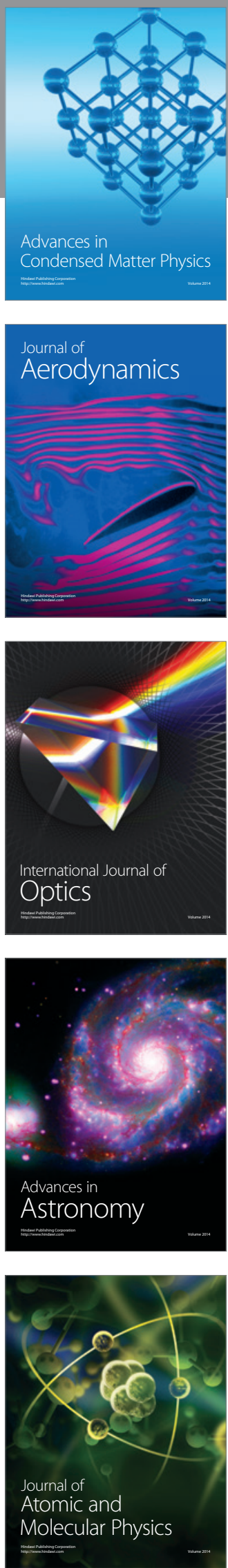